\title{
MEGARA-IFU detection of extended He II $\lambda 4686$ nebular emission in the central region of NGC 1569 and its ionization budget
}

\author{
Y. D. Mayya ${ }^{1 \star}$, E. Carrasco ${ }^{1}$, V.M.A. Gómez-González ${ }^{2}$, J. Zaragoza-Cardiel ${ }^{1,3}$, \\ A. Gil de Paz ${ }^{4}$, P. A. Ovando ${ }^{1}$, M. Sánchez-Cruces ${ }^{5}$, L. Lomelí-Núñez $^{1}$, \\ L. Rodríguez-Merino ${ }^{1}$, D. Rosa-González ${ }^{1}$, S. Silich ${ }^{1}$, G. Tenorio-Tagle ${ }^{1}$, \\ G. Bruzual ${ }^{2}$, S. Charlot $^{6}$, R. Terlevich ${ }^{1,7}$, E. Terlevich ${ }^{1}$, O. Vega ${ }^{1}$, J. Gallego ${ }^{4}$, \\ J. Iglesias-Páramo ${ }^{8,9}$, A. Castillo-Morales ${ }^{4}$, M.L. García-Vargas ${ }^{10}$, \\ P. Gómez-Alvarez ${ }^{10}$, S. Pascual ${ }^{4}$ and A. Pérez-Calpena ${ }^{10}$ \\ ${ }^{1}$ Instituto Nacional de Astrofísica, Óptica y Electrónica, Luis Enrique Erro 1, Tonantzintla 72840, Puebla, Mexico \\ 2 Instituto de Radioastronomía y Astrofísica, UNAM Campus Morelia, Apartado postal 3-72, 58090 Morelia, Michoacán, Mexico \\ 3 Consejo Nacional de Ciencia y Tecnología, Av. Insurgentes Sur 1582, 03940, Mexico City, Mexico \\ ${ }^{4}$ Dpto. de Física de la Tierra y Astrofísica, and Instituto de Física de Partículas y del Cosmos (IPARCOS), Fac. CC. Físicas, \\ Universidad Complutense de Madrid, Plaza de las Ciencias, 1, E-28040 Madrid, Spain \\ ${ }^{5}$ Aix Marseille Univ., CNRS, CNES, LAM, Marseille, France \\ ${ }^{6}$ Sorbonne Université, CNRS, UMR7095, Institut d'Astrophysique de Paris, F-75014, Paris, France \\ ${ }^{7}$ Institute of Astronomy, University of Cambrige, Cambridge CB3 OHA, UK \\ ${ }^{8}$ Instituto de Astrofísica de Andalucía, IAA-CSIC, Glorieta de la Astronomía s/n, E-18008 Granada, Spain \\ ${ }^{9}$ Estación Experimental de Zonas Áridas, CSIC, Carretera de Sacramento s/n, E-04120 Almería, Spain \\ ${ }^{10}$ FRACTAL S.L.N.E., Calle Tulipán 2, portal 13, 1A, E-28231 Las Rozas de Madrid, Spain
}

4 August 2020

\begin{abstract}
We here report the detection of extended He II $\lambda 4686$ nebular emission in the central region of NGC 1569 using the integral field spectrograph MEGARA at the 10.4-m Gran Telescopio Canarias. The observations cover a Field of View (FoV) of $12.5 \operatorname{arcsec} \times 11.3$ arcsec at seeing-limited spatial resolution of $\sim 15 \mathrm{pc}$ and at a spectral resolution of $\mathrm{R}=6000$ in the wavelength range $4330-5200 \AA$. The emission extends over a semi-circular arc of $\sim 40 \mathrm{pc}$ width and $\sim 150 \mathrm{pc}$ diameter around the super star cluster A (SSC-A). The $A_{\mathrm{V}}$ derived using Balmer decrement varies from the Galactic value of $1.6 \mathrm{mag}$ to a maximum of $\sim 4.5 \mathrm{mag}$, with a mean value of $2.65 \pm 0.60 \mathrm{mag}$. We infer $124 \pm 11$ Wolf-Rayet (WR) stars in SSC-A using the He II $\lambda 4686$ broad feature and $A_{\mathrm{V}}=2.3 \mathrm{mag}$. The $\mathrm{He}^{+}$ionizing photon rate from these WR stars is sufficient to explain the luminosity of the He II nebula. The observationally-determined total $\mathrm{He}^{+}$ and $\mathrm{H}^{0}$ ionizing photon rates, their ratio, and the observed number of WR stars in SSC-A are all consistent with the predictions of simple stellar population models at an age of $4.0 \pm 0.5 \mathrm{Myr}$, and mass of $(5.5 \pm 0.5) \times 10^{5} \mathrm{M}_{\odot}$. Our observations reinforce the absence of WR stars in SSC-B, the second most massive cluster in the FoV. None of the other locations in our FoV where He II $\lambda 4686$ emission has been reported from narrow-band imaging observations contain WR stars.
\end{abstract}

Key words: stars: emission-line - galaxies: star clusters - galaxies: individual (NGC 1569)

^ Email: ydm@inaoep.mx

Availability of large telescopes equipped with integral field spectrographs has enabled the creation of maps of

(c) 0000 The Authors 
He II $\lambda 4686$ nebular emission in nearby star-forming galaxies (e.g. Kehrig et al. 2015, 2018). Given that only photons shortward of $228 \AA(54 \mathrm{eV})$ can doubly ionize helium, the He II $\lambda 4686$ line provides a ground-based tool to trace the hard part of the ultraviolet (UV) spectrum. High mass stars, especially during their short-duration Wolf-Rayet (WR) phase, are the most common sources that emit these hard UV photons (Schaerer 1996). The UV flux emerging from a starburst region depends both on the hardness of the UV spectrum of WR stars as well as on the number of WR stars present. Because of the presence of an expanding atmosphere, models incorporating specialized line-blanketing treatment are required in order to quantitatively predict the flux of hard UV photons emitted by the WR stars. The emergent spectra in models incorporating such treatment (e.g. Hillier \& Miller 1998; Gräfener, Koesterke \& Hamann 2002) are found to be softer as compared to earlier models that did not take into account opacities from metals (Schmutz, Leitherer \& Gruenwald 1992). On the other hand, the number of WR stars and the duration of the WR phase in a starburst region depend on metallicity (e.g. Maeder \& Meynet 1989; Schaller et al. 1992; Bressan, Chiosi, Fagotto 1994; Chen et al. 2015), stellar rotation (Meynet \& Maeder 2005) and the stellar multiplicity (Eldridge et al. 2017). The calculated rate of $\mathrm{He}^{+}$ionizing photons at different metallicities depends critically on the mass-loss recipes used during massive star evolution. The general trend from the different set of currently available codes is a decrease in the expected luminosity of the He II $\lambda 4686$ line with decreasing metallicity.

The spectra from Sloan Digital Sky Survey (SDSS) have enabled the detection of the relatively faint He II $\lambda 4686$ line in large samples of star-forming galaxies (e.g. Shirazi \& Brinchmann 2012). These studies find that the observed He II $\lambda 4686 / \mathrm{H} \beta$ intensity ratio does not drop at low metallicities. In fact, recent studies find the ratio to be increasing as the metallicity decreases (Schaerer, Fragos \& Izotov 2019). Furthermore, these low-metallicity He II $\lambda 4686-$ emitting galaxies often show only a weak or no evidence of the presence of WR stars (Shirazi \& Brinchmann 2012). Thus, questions have been raised on the WR stars as the sole source of ionization of $\mathrm{He}^{+}$(Plat et al. 2019). Alternative mechanisms such as hard radiation from high-mass stars in binaries (Eldridge et al. 2017), shocks from supernova remnants (Garnett et al. 1991; Dopita \& Sutherland 1996) and high-mass X-ray binaries (HMXB; Schaerer, Fragos \& Izotov 2019; Kojima, et al. 2020) are often invoked. Nearby low-metallicity systems offer an opportunity to address the $\mathrm{He}^{+}$ionization problem by enabling study of individual star-forming knots. In a detailed study of the metalpoor $\left(\mathrm{Z}=3-4 \% \mathrm{Z}_{\odot}\right)$ galaxy SBS 0335 - 052E using MUSE, Kehrig et al. (2018) discard WR stars as the source of ionization and instead propose rotating metal-free stars or a binary population with $Z=10^{-5}$ and an extremely topheavy initial mass function (IMF) as the only plausible way of getting around the problem of the ionization budget. In a recent study Schaerer, Fragos \& Izotov (2019) find that the observed He II $\lambda 4686$ intensity in metal-poor star-forming galaxies can be naturally reproduced if the bulk of the $\mathrm{He}^{+}$ ionizing photons is emitted by the HMXB, whose number is found to increase with decreasing metallicity. X-ray binaries in a cluster appear only after the death of the most massive stars, and hence this scenario cannot explain the $\mathrm{He}^{+}$ ionization in young systems $(\mathrm{H} \beta$ equivalent widths (EWs) $\geqslant 200 \AA$ ), as illustrated by Plat et al. (2019).

A detailed analysis of the He II ionization budget problem has been carried out only in a handful of extreme metalpoor galaxies (e.g. Kehrig et al. 2011, 2018). Lack of observational data of individual massive stars at these low metallicities makes the predictions of population synthesis calculations heavily dependent on the theoretical assumptions. On the other hand, model calculations have been better calibrated at the LMC and solar metallicities. However, its detection requires sensitive observations, specially aimed at detecting faint emission lines. The newly available spectrograph MEGARA at the 10.4-m Gran Telescopio Canarias (GTC), equipped with Integral Field Unit (IFU) at spectral resolution $\sim 6000$ has the capability of detecting and mapping the faint emission lines from extragalactic nebulae (Gil de Paz et al. 2020). In order to exploit this capability, we carried out MEGARA observations of NGC 1569, a dwarf galaxy with a gas-phase oxygen abundance close to that of the LMC $(12+\log (\mathrm{O} / \mathrm{H})=8.19$; Kobulnicky \& Skillman 1997). We mapped its central region, which is known to have extended $\mathrm{H} \alpha$ emission (Hodge 1974; Waller 1991; Hunter, Hawley \& Gallagher 1993). Throughout this study, we use a distance of $3.1 \mathrm{Mpc}$, measured using Hubble Space Telescope (HST) observations of the tip of the red giant branch (Grocholski, et al. 2012). This distance is $\sim 10 \%$ smaller than that obtained by Grocholski et al. (2008) using an earlier analysis of the same dataset, but is still considerably higher than the 2.2 Mpc distance (Israel 1988) that was being routinely used in studies prior to the Grocholski et al. (2008) work.

NGC 1569 is among the nearest galaxies that harbours young superstellar clusters (SSCs) that are as massive as the Galactic globular clusters. Its most massive SSCs, called A and $\mathrm{B}$, are estimated to have dynamical masses of $4.1 \times$ $10^{5} \mathrm{M}_{\odot}$ (Ho \& Filippenko 1996) and $6.2 \times 10^{5} \mathrm{M}_{\odot}$ (Larsen et al. 2008), respectively (after rescaling the masses to the currently used distance of $3.1 \mathrm{Mpc}$ for this galaxy from Grocholski, et al. 2012). These two SSCs are at the high-mass end of a population of around $50 \mathrm{SSCs}$, whose ages range from a few million to several hundred million years (Hunter et al. 2000). The $\mathrm{H} \alpha$ morphology of the galaxy is dominated by $\mathrm{H}_{\text {II }}$ regions (Waller 1991), large-scale shells and superbubbles (Hunter, Hawley \& Gallagher 1993; Westmoquette et al. 2008), with the brightest $\mathrm{H}_{\text {II }}$ region in this galaxy associated to the star forming complex surrounding cluster 10 of Hunter et al. (2000) that lies 105 pc $(7$ arcsec) to the east of SSC-A, and is outside our FoV. Extended X-ray emission is detected in the galaxy, most of which coincides with the $\mathrm{H} \alpha$ bubbles (Martin et al. 2002; Sánchez-Cruces et al. 2015). Giant molecular clouds have been detected, but none of them are associated to SSCs A and B (Taylor et al. 1999). The largest $\mathrm{CO}$ cloud complex in the galaxy lies to the east of cluster 10 outside our FoV.

Long-slit spectroscopic observations have established the presence of WR stars in SSC-A, whereas no WR stars have been detected in SSC-B (González-Delgado et al. 1997). Using narrow-band HST imaging observations with the F469N filter, Buckalew et al. (2000) have inferred the presence of WR features from 5 SSCs, including SSC-A, and 7 individual stars, and nebular emission from three additional point sources. Kobulnicky \& Skillman (1997) reported faint He II nebular emission at some of the locations along the 
longslits they had used. However, no extended He II $\lambda 4686$ nebular emission has been yet detected in this galaxy. In this paper, we present our results obtained from spectral mapping of the central zone that includes both the SSCs A and $\mathrm{B}$.

In Section 2, we describe the observations and the data reduction. Techniques for separating the He II $\lambda 4686$ emission lines of stellar origin from nebular origin are also detailed in this section. In Section 3, we present the maps in these two components and compare them with the maps available at the $H S T$ resolution. Then, Section 4 deals with the calculation of the number of WR stars and the ionizing photon rate of doubly ionized helium. The ionization budget is discussed in Section 5, and our conclusions are given in Section 6. Measured data in individual fibre spectrum are presented in an appendix.

\section{OBSERVATIONS, REDUCTION AND DATA ANALYSIS}

\subsection{Observations}

Spectroscopic observations of the central part of NGC 1569 $(\mathrm{RA}=04: 30: 48.5, \mathrm{DEC}=+64: 50: 55.5)$ were carried out using the recently available MEGARA instrument at the 10.4-m GTC. MEGARA provides multi-object and integral field spectroscopy at low, medium and high spectral resolutions $R_{\mathrm{FWHM}} \equiv \lambda / \Delta \lambda \sim 6000,12000$ and 20000, respectively, in the visible wavelength interval from 3650 to $9700 \AA$, through 18 spectral configurations $(\Delta \lambda=$ Full Width at Half Maximum (FWHM) of a line). The IFU, also named Large Compact Bundle (LCB), covers an area on the sky of $12.5 \operatorname{arcsec} \times 11.3$ arcsec with 567 fibres for a spaxel size of 0.62 arcsec. To perform simultaneous sky subtraction, the IFU fibres are supplemented by another 56 fibres distributed in 8 mini-bundles of 7 fibres, located at the edge of the field at distances from 1.7 arcmin to 2.5 arcmin from the centre of the LCB. The complete set of 623 fibres is mounted in the LCB spectrograph pseudo-slit. Additionally, a set of robotic positioners host 92 mini-bundles of 7 fibres each, also for a spaxel size of 0.62 arcsec, allowing observations in a field of view $(\mathrm{FoV})$ of 3.5 arcmin $\times 3.5$ arcmin in the multi-object spectrograph (MOS) mode. These 644 fibres are arranged in a different pseudo-slit interchangeable with the LCB pseudoslit. For a complete description of MEGARA performance at GTC and the first scientific results obtained during the instrument commissioning, see Carrasco et al. (2018) and Gil de Paz et al. (2018, 2020).

The observations reported here were carried out as part of MEGARA guaranteed time on 2019 February $9^{\text {th }}$ in queue mode under dark sky and photometric conditions. The data were obtained using the IFU mode in combination with the LR-B volume phase holographic grating, centred at $4800 \AA$, covering the spectral range from $\sim 4330$ to $5200 \AA$ for a resolution of $\mathrm{FWHM}=0.78 \AA$ with a reciprocal dispersion of $0.21 \AA /$ pixel. Three $1200 \mathrm{~s}$ exposures were taken at airmasses between $1.25-1.30$, and seeing $\sim 0.9$ arcsec. For flux calibration the spectroscopic standard star HR5501 was observed in the same setup as for NGC 1569. Bias, flat field and arc lamp images were also obtained as part of the data package.

\subsection{Primary Data Reduction}

The primary reduction of the dataset was carried out using the standard MEGARA data reduction pipeline (DRP; Pascual et al. 2018). As described above, MEGARA/IFU data consist of 623 spectra corresponding to 567 object and 56 sky fibres, arranged into groups of multiple minibundles. In the spectral image, these groups are separated by gaps and have progressively smaller number of minibundles as they were built to reconstruct the curvature of the pseudo-slit at the focal plane of the MEGARA spectrograph. Flat-field images are used to trace the locus of each of the 623 spectra using an automatic routine. The routine fits simultaneously 623 Gaussians every 200 columns and then interpolates the parameters of the Gaussian for each spectral pixel. With this information, the routine generates a weight map for every fibre that is applied to the data in order to perform the extraction. The procedure allows correcting the flux of each fibre for cross-talk contamination from adjacent fibres to a level of a few per cent, depending on the quality of the spectrograph focus during the observation and the shape of the spectral PSF for each wavelength and fibre.

A shift along the pseudo-slit axis of the trace locus with dome temperature has been noticed, which needs a correction of the locus for each observed frame. We used sky lines to measure interactively the shifts, which are found to be $\sim 2$ pixels for the three spectral images used in this work. The final product of the DRP is a wavelength-calibrated, sky-subtracted 2D spectral image. This image contains 623 spectra, one spectrum corresponding to each fibre. The standard star is also reduced in an identical fashion. Spectra of all fibres containing the standard star are summed to extract a $1 \mathrm{D}$ spectrum, which is used to obtain the flux sensitivity curve using the IRAF ${ }^{1}$ routines for this purpose. The resulting sensitivity curve is used to obtain the flux-calibrated $2 \mathrm{D}$ images in selected spectral lines and the $3 \mathrm{D}$ data cube.

In Fig. 1, we show spectra of 4 fibres illustrating the detection of broad (top two panels) and narrow (bottom two panels) He II $\lambda 4686$ features. The Balmer lines $\mathrm{H} \beta$ and $\mathrm{H} \gamma$, and the $[\mathrm{OIII}] \lambda 5007 \AA$ line are also indicated.

\subsection{Line maps, data cube and astrometry}

The positions of the fibres in the image plane with respect to the image centre are given in millimeters in the image headers. The $2 \mathrm{D}$ spectral image is converted into a $3 \mathrm{D}$ data cube using these fibre coordinates. A plate scale of $1.212 \operatorname{arcsec~mm}{ }^{-1}$ and a fibre diameter of $0.62 \operatorname{arcsec}$ were used to transform the image coordinates from cartesian to equatorial systems. To create a map at any sampled wavelength (or a selected band corresponding to a line or a continuum region), we deposited the flux at that wavelength into hexagonal areas (the hexagonal spaxel is inscribed in a circle of 0.62 arcsec diameter), which correctly simulates the FoV of each fibre on the sky. Alternatively, to visualize extended emission, we also created smooth images by depositing the flux of a fibre into a highly oversampled

1 IRAF is distributed by the National Optical Astronomy Observatories, which are operated by the Association of Universities for Research in Astronomy, Inc., under cooperative agreement with the National Science Foundation. 


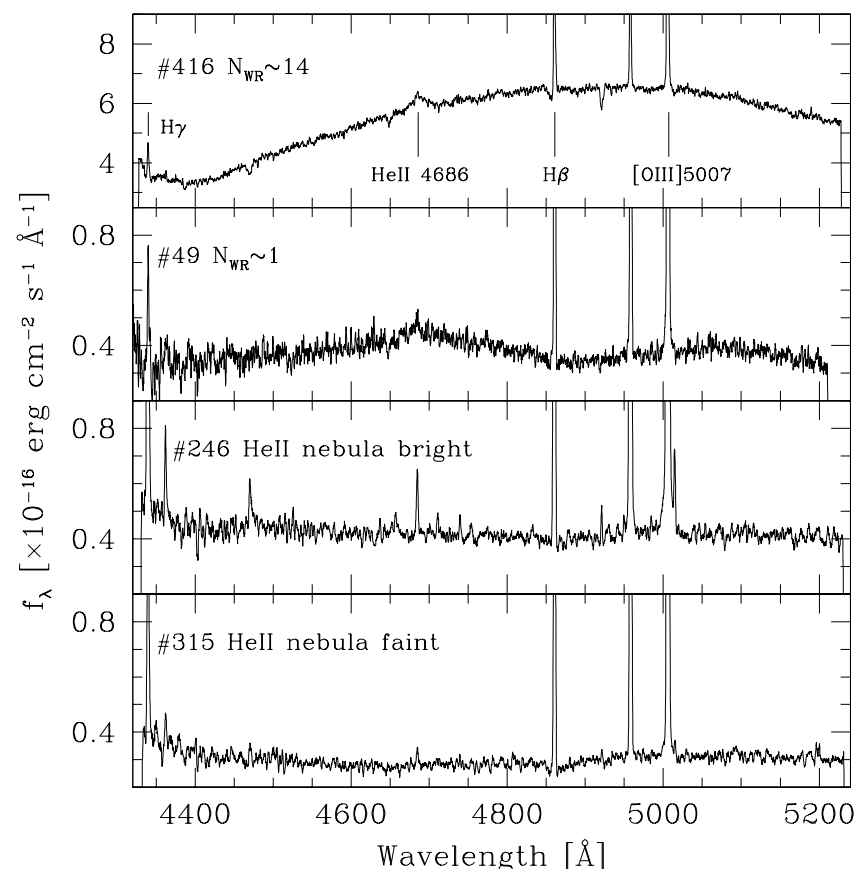

Figure 1. Spectra of individual fibres selected to illustrate broad emission at He II $\lambda 4686$ corresponding to WR stars (top two panels) and narrow He II $\lambda 4686$ from ionized nebula (bottom two panels). Four emission features are marked in the top-most spectrum. Fibre\#416, which belongs to SSC-A, illustrates the case of a spectrum containing multiple WR stars, whereas fibre\#49 illustrates the spectrum of a marginal detection of a WR star. Fibres\#246 and \#315 are examples of zones with bright and faint nebular He II $\lambda 4686$ line, respectively.

pixel (size $=0.021$ arcsec) and then convolving it with Gaussian kernel of $\sigma=$ half the fibre size $(0.31$ arcsec). Finally, the World Coordinate System header parameters were updated to force the RA and DEC of SSC-A to their values $(\mathrm{RA}=04: 30: 48.233, \mathrm{DEC}=+64: 50: 58.59)$ in the GAIA-DR2 system. After creating the image, we measured a mean coordinate error of $\sim 0.3$ arcsec for different sources in the resulting image.

We used the QFitsView facility (Ott 2012) for a quick visualization of the datacube and inspection of spectral features associated to known structures in the image. However, all analysis were carried out on the spectrum of an individual fibre or a sum of spectra of neighbouring fibres associated to a physical source. A datacube from the $2 \mathrm{D}$ spectral data was constructed using MEGARARSS2CUBE ${ }^{2}$, whereas the generation of line maps and their astrometry, extraction of spectra for physical regions and measurement of line fluxes were all carried out using our own scripts ${ }^{3}$ in the IRAF environment.

We calculated the error as the $1-\sigma$ deviation, $\sigma_{l}$, on each measured line flux using the expression (Tresse et al.

2 MEgararss2CuBE is a tool written in python to convert MEGARA reduced dataproducts from the RSS format obtained with megaradrp to a more user-friendly 3-D datacube, available as a repository in GitHub: https://github.com/javierzaragoza/megararss2cube

3 MEGARA-related IRAF scripts are available on request to the first author
1999):

$$
\sigma_{l}=\sigma_{c} D \sqrt{\left(2 N_{\mathrm{pix}}+\frac{E W}{D}\right)},
$$

where $D$ is the spectral dispersion in $\AA$ per pixel, $\sigma_{c}$ is the mean standard deviation per pixel of the continuum, $N_{\text {pix }}$ is the number of pixels covered by the line, and $\mathrm{EW}$ is the equivalent width of the measured line. We used the value of FWHM to substitute for $N_{\text {pix }}$.

\subsection{Ancillary Data}

We used HST images in F555W (ACS/HRC), F814W (ACS/WFC), F658N (ACS/WFC) and F469N (WFPC2) bands to associate our spectra to known structures in the image. All the HST images were brought to GAIA-DR2 coordinate system using the GAIA stars in the HST images. The first two images allow us to locate the stars and clusters, whereas the latter two trace the $\mathrm{H} \alpha$ and He II $\lambda 4686$ emitting sources, respectively. We used the identifications of the SSCs by Hunter et al. (2000) and WR and nebular He II sources by Buckalew et al. (2000). We also used the Chandra/ACIS $\mathrm{X}$-ray image in the $0.2-10 \mathrm{keV}$ band from Sánchez-Cruces et al. (2015) in order to locate the point and diffuse X-ray sources with respect to the optical sources.

\section{DETECTION OF WR AND NEBULAR He II $\lambda 4686$ EMISSION}

A simple visual inspection of the 2-D spectral image suggested the presence of a feature around $4686 \AA$ in many fibre spectra. Gaussian profile fitting of this feature suggested that in most cases, the feature is narrow with FWHM 1$2 \AA$, comparable or slightly above the resolution of the spectrograph. In some other fibres, the feature is broad with FWHM $>6 \AA$ A. These narrow and broad components are illustrated in the bottom two and top two spectra in Fig. 1, respectively. The narrow feature is easily identified as the He II $\lambda 4686$ emission line from the ionized nebula, whereas the broad component is identified as the blue bump (BB), which is the distinguishing characteristic of WR stars. The BB is a broad spectral feature between 4600 to $4700 \AA$, and consists of broad lines of $\mathrm{He}$ and/or $\mathrm{N}$ ions from nitrogen-rich WR stars (WN-types), or He and/or C ions from carbon-rich WR stars (WC-types). Among these, the prominent $\mathrm{N}$ and $\mathrm{C}$ lines are $\mathrm{N}$ III $\lambda \lambda 4634 / 41, \mathrm{~N}$ v $\lambda \lambda 4603 / 20$, C III $\lambda \lambda 4647 / 66$ and $\mathrm{C}$ IV $\lambda 4658$. WC stars, and hence lines from $\mathrm{C}$ ions, are generally absent in low-metallicity environments (see e.g. figure 8 in López-Sánchez \& Esteban 2010). Additionally, nebular lines He I $\lambda 4713$, He II $\lambda 4686$ and [Fe III] $\lambda 4658$ can also contribute to the BB. Some spectra showed both narrow and broad He II $\lambda 4686$ features, which required an analysis using multi-Gaussian fitting for the recovery of each component.

Another factor that affects the measurement of fluxes of relatively faint lines is determining the precise shape of the continuum, which requires a signal-to-noise ratio (SNR) of at least 10 in the continuum on either side within $\sim 100 \AA$ of the line of interest. In most continuum-weak fibres this condition is not met. For example, the SNR of the continuum in the bottom three spectra in Fig. 1 is $\sim 5$ at $4400 \AA$ to $\sim 9$ at 

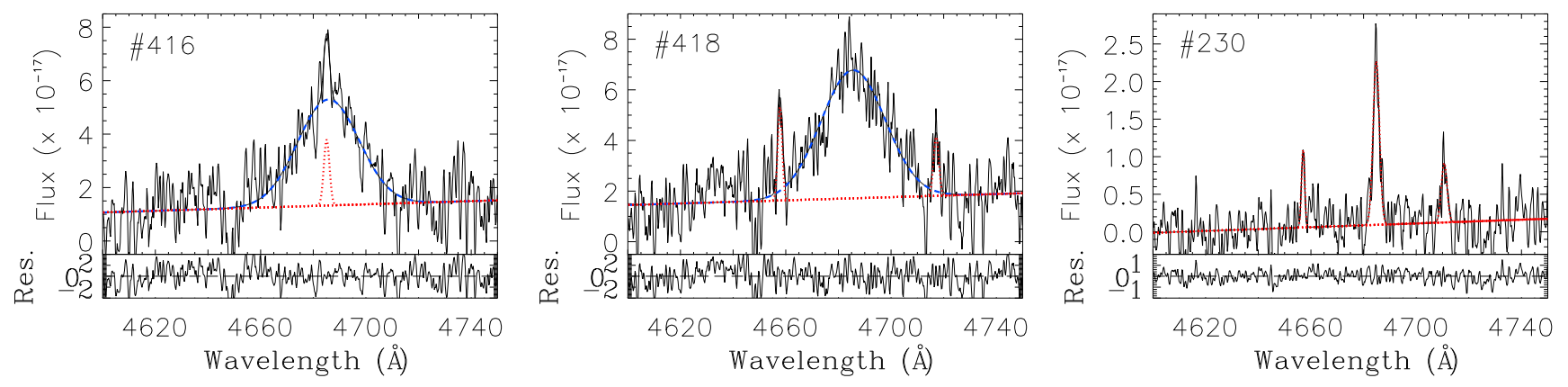

Figure 2. Multi-Gaussian fits to the blue bumps of individual fibres: broad He II $\lambda 4686$ line (blue), narrow He II $\lambda 4686$ nebular line (red) are shown for 3 representative fibre spectra that require: both the broad and narrow components (left; fibre\#416), only the broad component (middle; fibre\#418), and only the narrow component (right; fibre\#230). The fitted continuum is shown by the red straight line, and the residuals of the fit are shown below each fit. The fluxes in the fitted spectra as well as in the residual are in units of $10^{-17} \mathrm{erg} \mathrm{cm}^{-2} \mathrm{~s}^{-1} \AA^{-1}$. The middle and right spectra show additional narrow lines that we attribute to [Fe III] $\lambda 4658$ and He I $\lambda 4713$ nebular lines.

$4800 \AA$. On the other hand, the top spectrum, which belongs to SSC-A, has a minimum SNR of 23 at $4400 \AA$, increasing to 43 at $4800 \AA$ A. Stellar absorption lines, most of which are reported by González-Delgado et al. (1997) for SSC-A, can be seen in the top-most spectrum.

\subsection{Multi-component Gaussian decomposition of the blue bump}

It is well established that the individual lines that contribute to the BB can be extracted from multi-component Gaussian decomposition technique (see e.g. Brinchmann, Kunth \& Durret 2008). We hence carried out multi-Gaussian decomposition fittings using a custom-made code which uses the IDL routine LMFIT $^{4}$ (see Gómez-González et al. 2020). Before fitting multiple Gaussians, a continuum level is defined for each spectrum in a two-step procedure. First, a large-scale continuum is defined for each spectrum using the task continuum in IRAF, by fitting a high-order polynomial (spline3, order=11) passing through carefully chosen line-free bins in the entire wavelength range of the observed spectrum. Second, any residual local continuum around the $\mathrm{BB}$ is accounted for by linearly interpolating line-free zones on either side of the BB. Two of the 3 parameters, the peak intensity $I_{0}$ and the line width $\sigma$ that define each Gaussian, were left free. Any line with a FWHM $>6 \AA$ is defined as a broad line associated to a WR star. The third parameter, $\lambda_{0}$, is assigned to the rest wavelength of one of the expected lines from WR stars. The fitting program is executed interactively, where the bright nebular lines are fitted first, followed by the He II broad component. Residuals are examined for a peak near any of the expected line wavelength. If present, a second broad line is fitted and residuals are re-examined. While fitting this second line, the $\sigma$ and $I_{0}$ of the first line were left free. The process continues examining the residuals and adding a new line until the residual flux is less than 3 times the root-mean-square (RMS) noise of the spectrum. In the iterative process, any faint nebular lines are fitted, if

4 The LMFIT function (lmfit.pro) does a non-linear least squares fit to a function with an arbitrary number of parameters. It uses the Levenberg-Marquardt algorithm, incorporated in the routine mrqmin of Numerical Recipes in C (Press et al. 1992). needed. The method is able to recover the He II $\lambda 4686$ components from WR (broad) and nebula (narrow) even when both are present in a single spectrum. Fig. 2 illustrates the multi-Gaussian fits for spectra from 3 individual fibres that require both a broad and a narrow He II $\lambda 4686$ line (left), only a broad line (middle) and only the narrow line (right). None of our fibre spectra showed broad lines from nitrogen or carbon ions. A feature is recovered in many fibres at $\lambda=4658 \AA$ (see the spectra in the middle and right panels), which matches the wavelength of the C IV $\lambda 4658$ (broad) feature. However, the width of this line is comparable to that of nebular lines, and hence we identify this feature with the nebular (narrow) line [Fe III] $\lambda 4658$.

We used the fitting program to automatically identify broad and narrow He II $\lambda 4686$ features in each of the 567 spectra. As mentioned above, the flux at the peak of the fitted Gaussian should be at least 3 times the noise level for it to be considered a detection. The narrow component, which is of nebular origin, is detected in 262 of these fibres and the broad component, in 50. Twenty five of the spectra showing a broad component have an associated narrow component, whereas the remaining 25 show only a broad component.

\subsection{Location of fibres with WR features}

In Fig. 3, we show the positions of all the 50 individual fibres with broad He II $\lambda 4686$ detections (hexagons ${ }^{5}$ ), overlaid on an RGB colour-composite image formed by HST images in F814W, F555W and F469N filters, shown as red, green and blue colours, respectively. This set of images was chosen in order to highlight the candidate WR stars, which are expected to be continuum-bright sources with He II $\lambda 4686$ excess. The red and green images having been taken using broad bands, trace continuum sources, whereas the blue image traces sources that have contribution from emission lines in the $4650-4720 \AA$ range. The He II $\lambda 4686$ line is the most likely contributor to this image, but as the spectra in Fig. 2 illustrate, nebular lines [Fe III] $\lambda 4658$ and He I $\lambda 4713$ could also contribute in this filter. Thus, blueish-looking

5 See Fig. A1 in the appendix for the identification numbers of each fibre. 


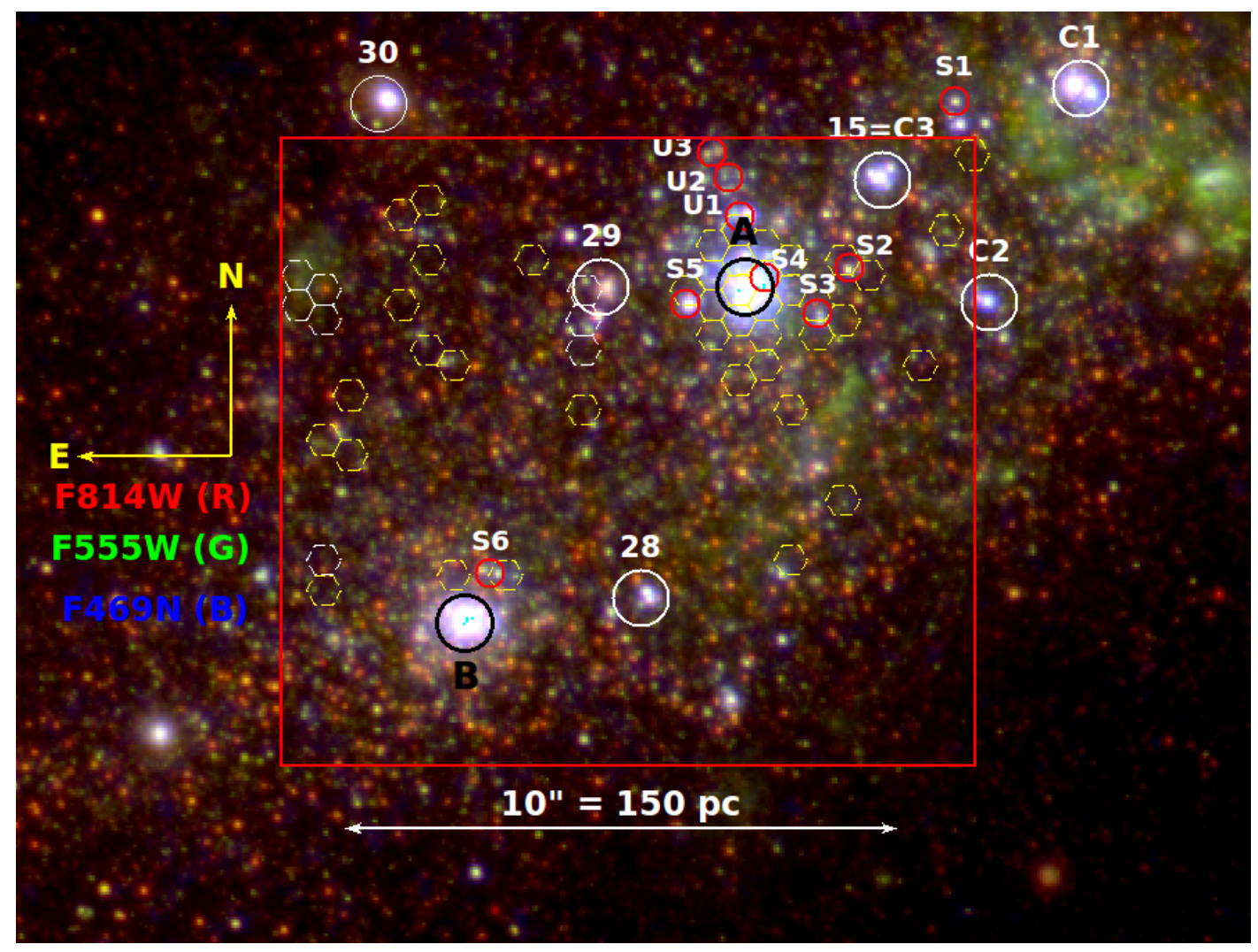

Figure 3. A colour-composite RGB image of the central starburst zone of NGC 1569. The image is formed by combining HST F814W, F555W and F469N band images, as red, green and blue components, respectively. MEGARA/IFU FoV (12.5 arcsec $\times 11.3$ arcsec; red rectangle) and image orientation (compass) are shown. The locations of the fibres where we detected a broad He II $\lambda 4686$ component are shown by hexagons. The prominent objects in the FoV are identified. These include the two massive clusters SSC-A and SSC-B, and four other clusters (15, 28, 29 and 30) from Hunter et al. (2000). WR sources identified by Buckalew et al. (2000) using the displayed HST F469N image, which intercepts the He II $\lambda 4686$ line, are shown. These include four clusters with WR stars (C1, C2, C3 and C4) and six WR stars (S1, S2, S3, S4, S5 and S6). Three sources (U1, U2 and U3) classified as of unknown origin by Buckalew et al. (2000) are also shown. The He II $\lambda 4686$ broad feature is detected in 18 adjacent fibres associated to SSC-A. The circles enclosing cluster candidates have a diameter of 1 arcsec, and hexagons have an equivalent diameter of 0.62 arcsec.

continuum-bright sources in this image are the likely WR candidates. Buckalew et al. (2000) used the F469N image in combination with continuum and nebular images to identify WR candidate sources. They classified these as cluster, stellar, and unknown origin sources, and named them as $\mathrm{C} 1$ to C4, S1 to S6, and U1 to U3 sources, respectively, which are all identified in the figure. In addition, we identified stellar clusters catalogued by Hunter et al. (2000) (numbers 15, 28, 29 and 30) and the two well-known SSCs A and B in the figure. Cluster C4, which lies just outside our FoV and is identified as cluster 10 by Hunter et al. (2000), is the ionizing cluster of the brightest $\mathrm{H}_{\text {II }}$ region in this galaxy.

The presence of the He II $\lambda 4686$ broad component is the characteristic signature of a WR star. However, given that our observations were carried out at $\sim 0.9$ arcsec seeing and the fibres cover a diameter of 0.62 arcsec, several adjacent fibre spectra should also show up the broad feature in order to associate the inferred feature with the detection of a WR star. Out of the 50 fibres where we inferred He II $\lambda 4686$ broad component, 18 contiguous fibres are associated to SSC-A. Some of these fibres could be associated to candidate stellar sources S3, S4 and S5, which are located within the seeingconvolved image of SSC-A.
Of the remaining detections, we infer two locations with four (\#417, 419, 421, 423; on the top-left) and three (\#145, 149, 153; on the top-middle) contiguous fibres, associated to them. However, a careful scrutiny of the fibres associated to these locations revealed that these fibres are physically next to one of the 18 fibres belonging to SSC-A on the psuedoslit, and hence the detected broad bump is likely arising due to residual cross-talk from SSC-A. Hence, we ignore the detections in these seven fibres. The rest of the inferred He II $\lambda 4686$ broad component corresponds to 25 single-fibre detections, scattered all over the image. One of these detections (\#413) is due to cross-talk with SSC-A. Of the remaining detections, two coincide with the previously reported candidate stellar sources S2 and S6. If these single-fibre detections come from real sources, spectra extracted by summing spectra of fibres adjacent to the location of these fibres also should show a broad He II $\lambda 4686$ feature. We carried out the Gaussian decomposition of the BB with the specific purpose of detecting these broad features in spectra obtained by summing spectra of at least 3 fibres around the one where we detected the broad feature. None of these summed spectra around the 25 single-fibre detections showed a broad component. Uncertainty in defining the continuum is the likely 

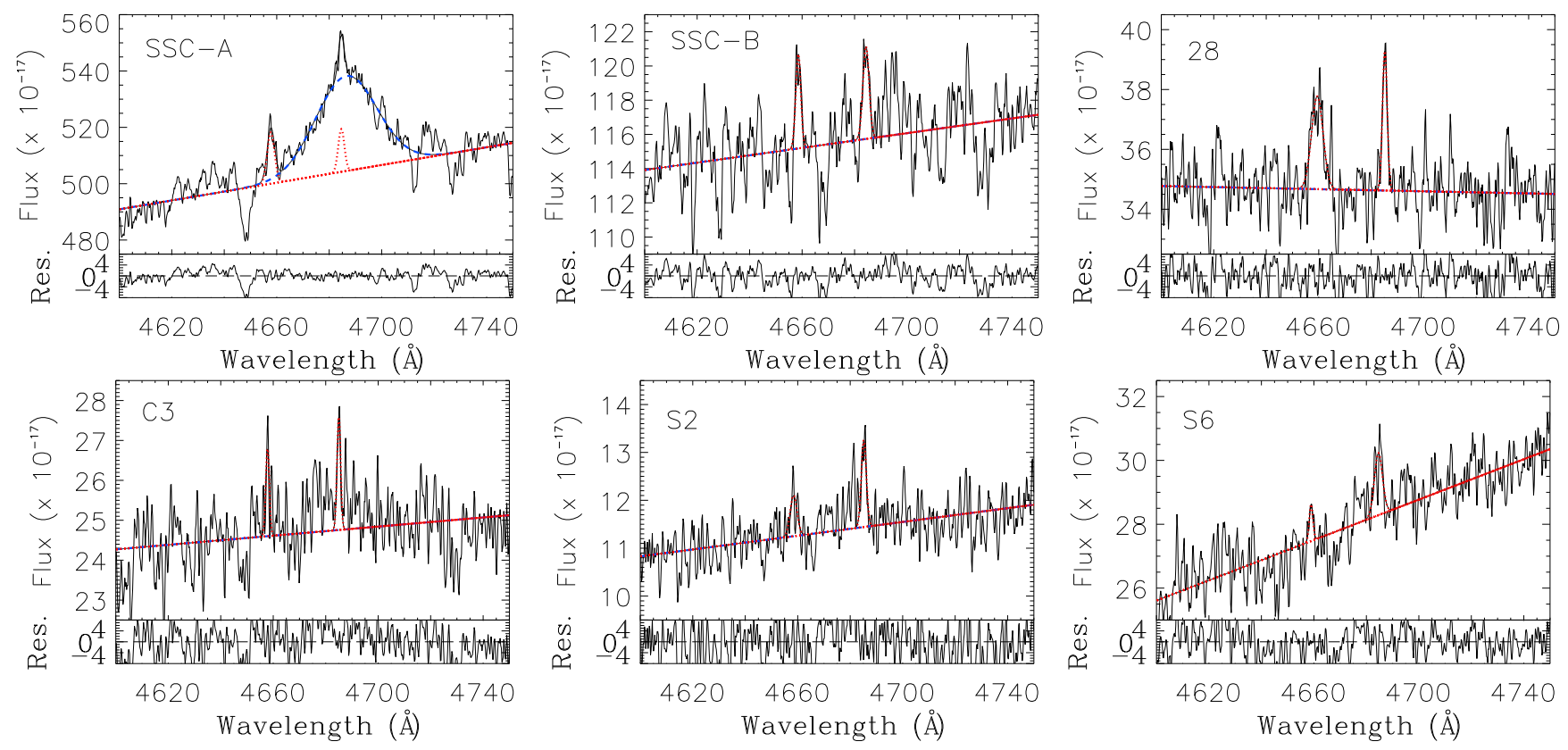

Figure 4. Multi-fibre summed spectra around the BB of SSC-A and other cluster and stellar sources in the FoV of MEGARA/IFU. No WR stars are detected in clusters SSC-B and 28. Star cluster C3 and stellar sources S2 and S6, where the presence of WR stars have been previously reported from the analysis of the HST F469N-band narrow-band images, only show nebular lines in our spectra. The fluxes are plotted in units of $10^{-17} \mathrm{erg} \mathrm{cm}^{-2} \mathrm{~s}^{-1} \AA^{-1}$, and the bottom panel of each plot shows the residual flux in the same units.

reason for the feature to vanish in summed spectra in spite of them being detected, i.e. the peak flux of the fitted broad (FWHM $>6 \AA$ ) Gaussian profile is $>3$ times the RMS error of the continuum, in individual fibre spectra. The spectrum for fibre \#49 in Fig. 1 is one such example. Deeper observations would be required to ascertain the nature of these sources. We consider these detections as marginal and in $\S 4.3$ we compare their fluxes to that expected from typical WNL-type WR stars.

The summed spectra allow us to understand the reasons why Buckalew et al. (2000) interpreted the observed excess emission in the $H S T / \mathrm{F} 469 \mathrm{~N}$ band in $\mathrm{C} 3, \mathrm{~S} 2$ and $\mathrm{S} 6$ as originating in WR stars. It may be recalled that imaging observations cannot distinguish between the narrow and broad components. Buckalew et al. (2000) assumed it to be a WR detection, if the position of a star or cluster coincides with the position where an excess flux is detected in the F469N band. This selection criterion does not contemplate the presence of extended He II $\lambda 4686$ nebular emission. As we discuss in detail below, we have detected extended He II $\lambda 4686$ nebular emission in the central zone of NGC 1569. Objects C3, S2 and $\mathrm{S} 6$ are stellar sources in which we detect narrow nebular He II $\lambda 4686$ and [Fe III] $\lambda 4658$ lines in our spectra (see the bottom three spectra in Fig. 4). The narrow lines are not originating in the stellar sources, and are instead part of the extended nebular emission. At the location of the three sources of unknown origin (U1, U2 and U3), we detect only narrow He II $\lambda 4686$ line; these sources are also part of the extended nebula.

Our spectral data are used to infer the WR population in known clusters in our FoV. Apart from SSC-A, where we already demonstrated the presence of WR stars, our FoV includes clusters SSC-B, 28, 29, and C3. Among these, pre- vious spectroscopic observations have already ruled out the presence of WR features in SSC-B (e.g. González-Delgado et al. 1997). No WR stars were inferred in F469N images by Buckalew et al. (2000) from sources 28 and 29, whereas C3 is a candidate WR cluster. We extracted spectra around all these sources, as well as for SSC-A (C4), by adding spectra of 7 fibres around the location of these clusters. Unfortunately, cluster 29 spectrum suffers from cross-talk problem as several of its fibres are adjacent to some of the fibres of SSC-A on the pseudo-slit. We hence did not carry out an analysis of search for WR stars in this cluster. Multi-Gaussian analysis was carried out on these summed spectra, which are shown in Fig. 4. These fits establish the presence of WR features in summed spectra of SSC-A, and their absence in SSC-B, 28 and C3. The figure also shows (last two panels) the results of the fits to the summed spectra for $\mathrm{S} 2$ and S6. Given the closeness of these sources to SSC-A and SSC-B, respectively, we summed spectra of only 3 adjacent fibres in these cases. The broad He II $\lambda 4686$ component is not recovered in these summed spectra.

\subsection{He II $\lambda 4686$ nebular morphology}

We now discuss the results obtained by muti-Gaussian fitting to recover the He II $\lambda 4686$ nebular line. Unlike the broad component, the narrow He II $\lambda 4686$ line, which is of nebular origin, is detected in nearly half of the total fibres. The fibre fluxes and locations are used to create a map, which is shown in Fig. 5 as the green component of the RGB image. The HST images in F658N ( $\mathrm{H} \alpha+$ continuum) and $\mathrm{F} 555 \mathrm{~W}$ bands are used for the red and blue components, respectively. These latter images help us to see the large-scale distribution of ionized gas and stars at the HST resolution. 


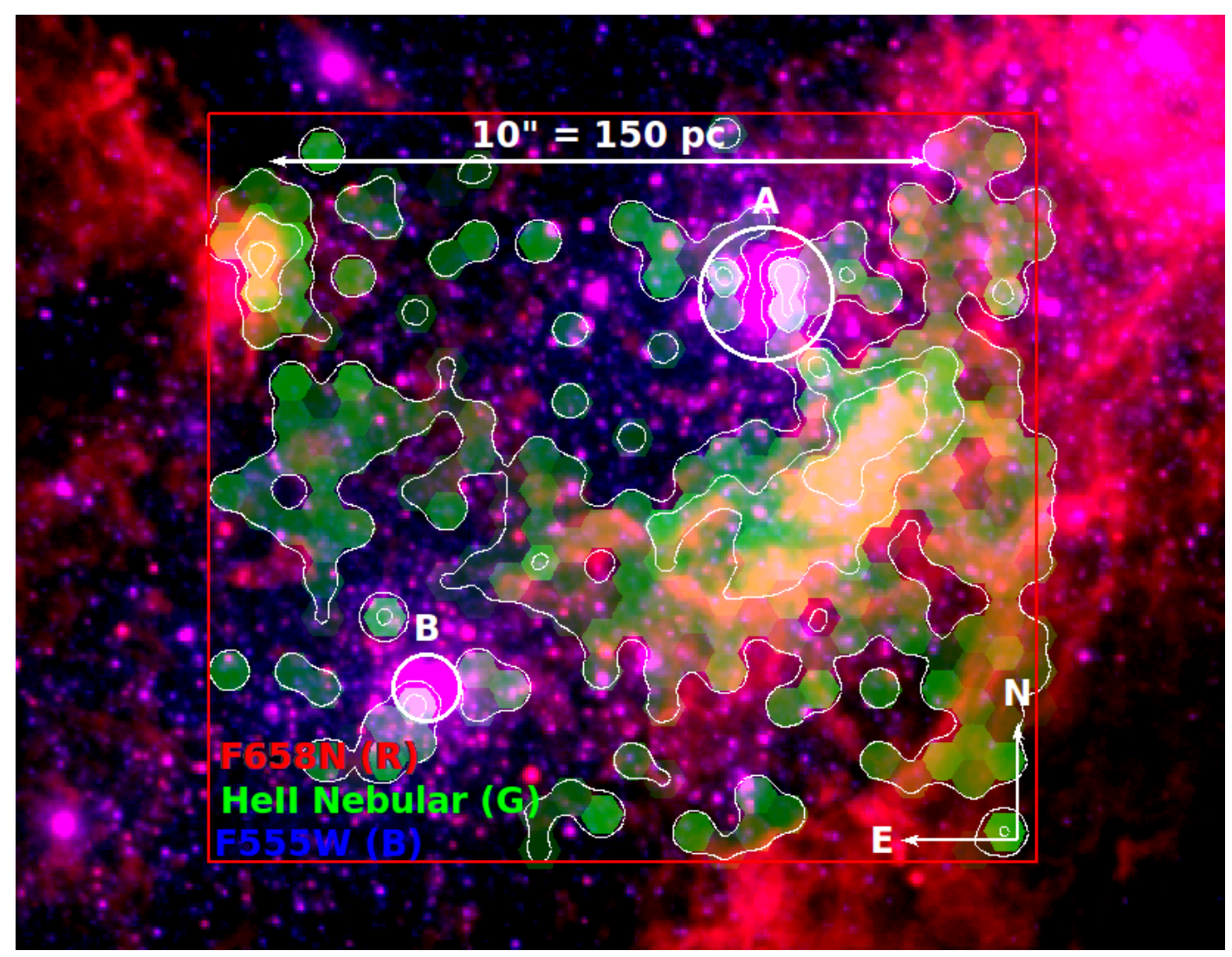

Figure 5. Colour-composite RGB image showing the morphology of the He II $\lambda 4686$ nebular emission (white contours englobing green hexagons) with respect to that of the H $\alpha$ emission ( $H S T$ F658N-band shown in red) and stellar continuum (HST F555W image shown in

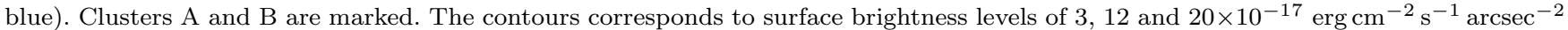
in the He II $\lambda 4686$ nebular line. The nebular emission is weak from SSCs A and B, and is distributed along a semi-circular arc with the most intense emission coming from a zone $\sim 40 \mathrm{pc}$ south-west of SSC-A.

Contours corresponding to He II $\lambda 4686$ nebular line surface brightness of 3,12 and $20 \times 10^{-17} \mathrm{erg} \mathrm{cm}^{-2} \mathrm{~s}^{-1} \operatorname{arcsec}^{-2}$ are shown.

Clearly the He II $\lambda 4686$ narrow emission is not confined to a few point sources, but instead is part of an extended nebula. This emission is distributed along a semi-circular arc of 150 pc $(10 \operatorname{arcsec})$ diameter. The brightest part of the He II $\lambda 4686$ nebula lies to the south-west of SSC-A at a distance of $\sim 40 \mathrm{pc}(2.5 \mathrm{arcsec})$. This brightest part is also the zone closest to SSC-A, where there is an increase in the $\mathrm{H} \alpha$ surface brightness, and corresponds to the $\mathrm{H}$ il region numbered 3 by Waller (1991). The nebular arc is widest near this zone, reaching $\sim 40 \mathrm{pc}$ width. The centre of the semicircular arc does not coincide with SSC-A, and instead is shifted to the east of it by $\sim 40 \mathrm{pc}$, roughly coincident with cluster 29. Unfortunately our FoV does not cover the northern part, hence we cannot conclude whether the observed arc is part of a complete circular nebula or not. There is no evidence for arc-like structure in the $\mathrm{H} \alpha$ image on the northern side, nor any of the ionized superbubbles (sb) identified by Sánchez-Cruces et al. (2015) match our He II $\lambda 4686$ emitting segment or its possible northern counterpart. This can be due to the lower sensitivity of Fabry-Pèrot images presented by Sánchez-Cruces et al. (2015) in comparison to the HST images. The He II $\lambda 4686$ emitting segment lies between the superbubbles classified as sb4, sb5, sb6 and sb7.
The He II $\lambda 4686$ nebular emission is weak in the immediate vicinity of SSC-A and SSC-B.

By mapping the He II $\lambda 4686$ nebular emission we can obtain the total flux in this line, and from this, the ionization requirements. The data need to be corrected for extinction from dust along the line of sight in the Galaxy as well as the dust in NGC 1569, before we can carry out these calculations. The availability of the extinction-sensitive Balmer lines $\mathrm{H} \beta$ and $\mathrm{H} \gamma$ in our dataset allows us to obtain the extinction from each fibre spectrum. We describe the procedure adopted for extinction correction in the next section.

\section{EXTINCTION-CORRECTED NUMBER OF WR STARS AND THE He II IONIZING PHOTON RATE}

In this section, we present the extinction map of the zone covered by our IFU observations, and obtain the He II $\lambda 4686$ luminosity of the broad and narrow lines, corrected for extinction. The luminosity in the broad component is used to calculate the number of WR stars inferred from our observations, whereas the luminosity in the narrow component is used to obtain the $\mathrm{He}^{+}$ionizing photon rate. We used the emission line intensities of Balmer lines $(\mathrm{H} \beta$ and $\mathrm{H} \gamma$ ) in our IFU spectra to create maps of the ionized nebula 
and extinction. We note that the stellar continuum of unresolved populations, in general, experiences lesser amount of attenuation as compared to that experienced by the ionized gas (Calzetti, Kinney \& Storchi-Bergmann 1994; Mayya \& Prabhu 1996). Hence, the number of WR stars derived after correcting the broad component luminosity for nebular extinction could be an over-estimate.

\subsection{Extinction map of the observed zone}

In $\S 3.1$, we described the procedure we followed for decomposing the narrow and broad components of the He II $\lambda 4686$ feature. We measured the fluxes and related quantities of $\mathrm{H} \beta$ and $\mathrm{H} \gamma$ nebular lines in each fibre spectrum using the single Gaussian fitting option of the IRAF task splot in batch mode. The line fluxes in each fibre are then transformed into maps using the procedure described in $\S 2.3$.

$\mathrm{H} \beta$ and $\mathrm{H} \gamma$ emission fluxes were used to obtain the extinction from each fibre spectrum, where both of these lines were detected, using the Balmer decrement method for case $\mathrm{B}$ recombination of a typical photoionized nebula $\left(T_{\mathrm{e}}=10000 \mathrm{~K}, n_{\mathrm{e}}=100 \mathrm{~cm}^{-3}\right.$; Osterbrock \& Ferland 2006) and the reddening curve of Cardelli, Clayton \& Mathis (1989). Only spectra with a SNR of at least 3 in both the lines are used. The $\mathrm{H} \beta$ line is detected at $\mathrm{SNR}>7$ in all the 567 fibres. This line is well-resolved with the FWHM of the fitted Gaussian varying between 1 and $2 \AA$. Some spectra clearly showed signs of more than one velocity component. In $86 \%$ of these spectra (487), the $\mathrm{H} \gamma$ line is also detected at $\mathrm{SNR} \geqslant 3$, enabling $A_{\mathrm{V}}$ measurements in all these spectra. The resulting values along with the errors are given in the last two columns of Table A1. However, all measurements are not reliable. A source of error in the derived $A_{\mathrm{V}}$ is the uncertain correction of the underlying absorption in $\mathrm{H} \beta$ and $\mathrm{H} \gamma$ lines. We used a uniform $1 \AA$ of correction in EW proposed by González-Delgado et al. (1997) for both the $\mathrm{H} \beta$ and $\mathrm{H} \gamma$ lines. The underlying absorption affects spectra at locations where we detected strong continuum, some of which belong to the WR stars. Thus, it is reasonable to use the $A_{\mathrm{V}}$ obtained for continuum-weak, or alternatively high EW, spectra for carrying out fibre-to-fibre $A_{\mathrm{V}}$ corrections. As $\mathrm{H} \gamma$ is the more critical of the two lines in $A_{\mathrm{V}}$ measurements, we used only those spectra that have $\operatorname{EW}(\mathrm{H} \gamma)>8 \AA$. This left us with the best 106 measurements of $A_{\mathrm{V}}$. The resulting $A_{\mathrm{V}}$ values are plotted against the $\mathrm{SNR}$ of the $\mathrm{H} \gamma$ line, which is the fainter of the two lines used for determining extinction, in Fig. 6. The estimated error on each measurement is shown, which is typically $\sim 0.5 \mathrm{mag}$. These error bars do not take into account errors introduced by the uncertain correction for the underlying stellar absorption, e.g. the $A_{\mathrm{V}}$ values would be $\sim 0.2 \mathrm{mag}$ higher or lower depending on no correction, or correction of $2 \AA$ (instead of the assumed $1 \AA$ ), respectively.

Devost, Roy \& Drissen (1997) obtained $A_{\mathrm{V}}$ using $\mathrm{H} \alpha$ and $\mathrm{H} \beta$ lines for 16 zones spread over the entire galaxy. They found all zones have $A_{\mathrm{V}} \geqslant 1.6 \mathrm{mag}$, with the minimum value corresponding to a zone in an ionized bubble, which is outside our FoV. They proposed that this minimum value corresponds to the extinction from the Galactic dust along the line of sight to NGC 1569. Grocholski, et al. (2012) obtained a marginally higher value $\left(A_{\mathrm{V}}=1.8 \mathrm{mag}\right)$ using an analysis of the red giant branch of the resolved stellar population in

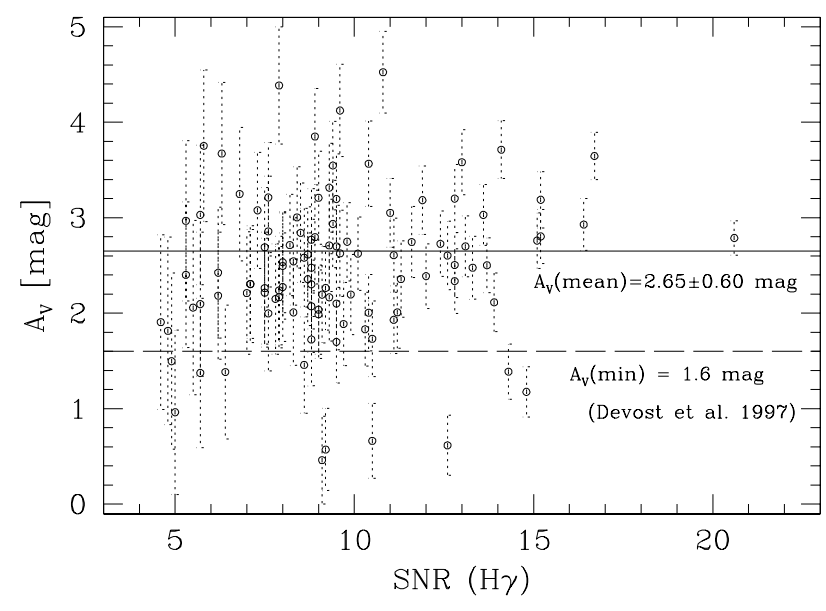

Figure 6. Visual extinction $A_{\mathrm{V}}$ obtained from the $\mathrm{H} \beta$ and $\mathrm{H} \gamma$ nebular lines is plotted against the $\mathrm{SNR}$ of the $\mathrm{H} \gamma$ line, which is the weaker of the two lines. Only 106 spectra for which the $\mathrm{EW}(\mathrm{H} \gamma)>8 \AA$ are considered for plotting. Among the plotted points, $90 \%$ lie above the dashed horizontal line, which corresponds to the minimum $A_{\mathrm{V}}$ in the entire galaxy found by Devost, Roy \& Drissen (1997). The mean value of our measurements is shown by the solid horizontal line. See $\S 4.1$ for further details.

the outer disk of the galaxy. There are many independent measurements of the Galactic extinction in the direction of NGC 1569. Using the line of sight HI observations, Burstein \& Heiles (1984) and Schlegel, Finkbeiner \& Davis (1998) obtained values of $A_{\mathrm{B}}=2.03 \mathrm{mag}$ (equivalent to $A_{\mathrm{V}}=1.55 \mathrm{mag}$ ) and $A_{\mathrm{V}}=2.3 \mathrm{mag}$, respectively. Israel (1988) and Origlia et al. (2001) analysed the UV spectra of NGC 1569 and suggested a foreground extinction of $A_{\mathrm{V}}=1.7 \mathrm{mag}$. More recently, Schlafly \& Finkbeiner (2011) obtained a value of $A_{\mathrm{V}}=1.9$ mag based on the colour excess of Sloan Digital Sky Survey (SDSS) stars that have spectroscopic data.

All these values, with the exception of one (Schlegel, Finkbeiner \& Davis 1998), are compatible with a Galactic extinction value of $A_{\mathrm{V}}=1.6 \mathrm{mag}$ proposed by Devost, Roy \& Drissen (1997) after taking into account measurement errors. We show this lower limit by a dashed line in Fig. 6 . Ninety per cent of our measurements are above this line. Nine of the 11 measurements below this line are consistent with $A_{\mathrm{V}}=1.6 \mathrm{mag}$ if we calculate $A_{\mathrm{V}}$ without any absorption correction. Thus, taking into account external errors, our data are in agreement with a minimum $A_{\mathrm{V}}=1.6 \mathrm{mag}$ proposed by Devost, Roy \& Drissen (1997). The rest of the H II regions observed by Devost, Roy \& Drissen (1997) have a mean value of $A_{\mathrm{V}}=2.26 \mathrm{mag}$, implying an average internal extinction of $0.66 \mathrm{mag}$ for NGC 1569. We obtain a mean $A_{\mathrm{V}}=2.65 \pm 0.60 \mathrm{mag}$ for 95 spectra having $A_{\mathrm{V}}>1.6 \mathrm{mag}$. This implies an average internal extinction of $1.05 \mathrm{mag}$ for the zone covered by our observations.

We used the $A_{\mathrm{V}}$ values obtained in these 95 fibres to create an extinction map, whose contours are shown in Fig. 7, overlaid on a nebular colour-composite image, formed by combining $\mathrm{H} \beta$, He II $\lambda 4686$ and $\mathrm{H} \gamma$ images as red, green and blue components, respectively. All the three nebular images are smoothed using a Gaussian kernel of 0.31 arcsec following the procedure described in $\S 2.3$. As the $\mathrm{H} \gamma$ to $\mathrm{H} \beta$ 


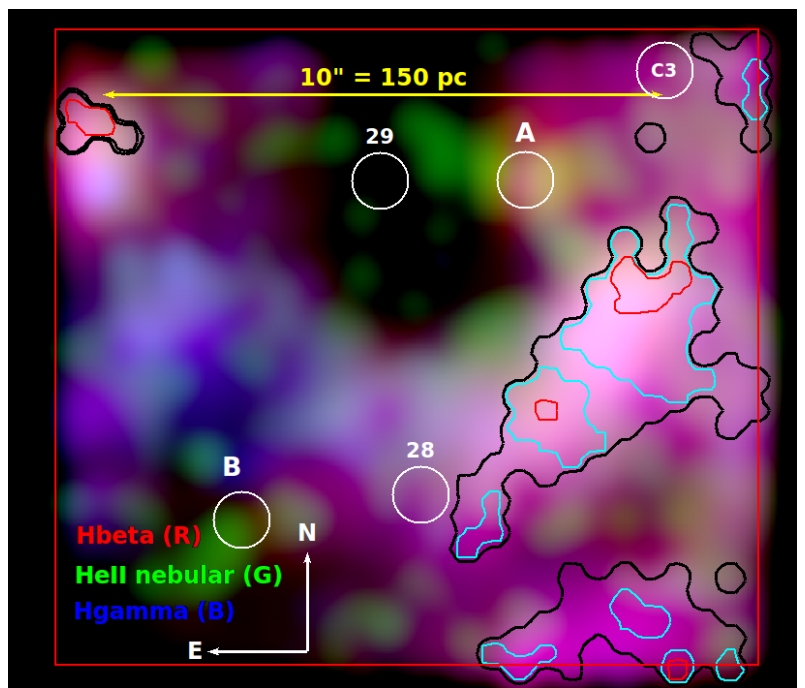

Figure 7. Extinction contours superposed on the MEGARA RGB nebular images. Smoothed images in $\mathrm{H} \beta$, He II $\lambda 4686$ and $\mathrm{H} \gamma$ are used as red, green and blue components, respectively. The contours corresponding to $A_{\mathrm{V}}=1.6,2.6$ and $3.6 \mathrm{mag}$ are shown in black, cyan and red colours, respectively. The noise in the measured fluxes in individual fiber spectra did not allow the determination of reliable $A_{\mathrm{V}}$ values outside the black contours, though emission is seen in this smoothed image. Known sources in the FoV are labelled. The zone with the highest He II $\lambda 4686$ nebular emission (south-west of SSC-A) is also the zone with the highest $A_{\mathrm{V}}$. The nebular emission follows a semi-circular arc of $\sim 150 \mathrm{pc}$ diameter.

ratio is sensitive to extinction, the colour in the image is expected to change from blue to red as extinction increases. The zone with the highest He II $\lambda 4686$ nebular surface brightness (south-west of SSC-A) is also the zone with the highest $A_{\mathrm{V}}$, reaching values $\sim 4$ mag. Extinction smoothly decreases from this point towards the south-east, reaching the Galactic values of $A_{\mathrm{V}}=1.6 \mathrm{mag}$ at the boundaries of the detected zone. High extinction is also inferred from the zone with weak nebular emission in the bottom-right corner of the image, and a zone at the top-left corner of the image. Nebular lines are weak in almost all continuum-bright regions, including in SSC-A, which prevented us from determining $A_{\mathrm{V}}$ for these sources. We have assigned the Galactic values of $A_{\mathrm{V}}=1.6 \mathrm{mag}$ to all regions outside the black contours, where $A_{\mathrm{V}}$ could not be reliably measured. Fig. 7 also allows us to compare the large-scale morphology of He II $\lambda 4686$ nebula (green) with that of the ionized gas traced by Balmer lines at the same resolution. The $\mathrm{H}_{\text {II }}$ gas is also distributed along the semi-circular arc traced by the He II nebula, both showing a clear hole in the central zone of their intensity distributions.

\subsection{Total He II ionizing photon rate}

The availability of an extinction map allows us to apply fibre-to-fibre extinction corrections. We used a uniform value of $A_{\mathrm{V}}=1.6 \mathrm{mag}$ to all spectra where $A_{\mathrm{V}}$ could not be measured reliably. Summing all these fluxes, we obtain a total He II $\lambda 4686$ flux of $9.13 \times 10^{-14} \mathrm{erg} \mathrm{cm}^{-2} \mathrm{~s}^{-1}$. This corresponds to a $\mathrm{L}\left(\mathrm{He}\right.$ II $\lambda 4686$ ) of $1.0 \times 10^{38} \mathrm{erg} \mathrm{s}^{-1}$ and a $\mathrm{Q}\left(\mathrm{He}^{+}\right)$ of $1.0 \times 10^{50}$ photon $^{-1}$ using the basic photo-ionization equation from Osterbrock \& Ferland (2006),

$$
\begin{aligned}
\frac{\mathrm{Q}\left(\mathrm{He}^{+}\right)}{\text {photon s}^{-1}} & =\frac{L(\mathrm{He} \mathrm{II} \lambda 4686)}{E_{\lambda 4686}} \times \frac{\alpha_{\mathrm{B}}\left(\mathrm{He}^{+}\right)}{\alpha_{\mathrm{eff}}(\mathrm{He} \mathrm{II} \lambda 4686)} \\
& =1.02 \times 10^{48} \frac{L(\mathrm{He} \mathrm{II} \lambda 4686)}{10^{36} \mathrm{erg} \mathrm{s}^{-1}}
\end{aligned}
$$

where $E_{\lambda 4686}$, is the energy of the He II $\lambda 4686$ photon, and $\alpha_{\mathrm{B}}$ and $\alpha_{\text {eff }}$ are case-B recombination coefficients. We used the $\alpha_{\mathrm{B}}$ and $\alpha_{\text {eff }}$ assuming reasonable $T_{\mathrm{e}}=10000 \mathrm{~K}$ and $n_{\mathrm{e}}=100 \mathrm{~cm}^{-3}$ for the nebula. The calculated $\mathrm{Q}\left(\mathrm{He}^{+}\right)$would change by less than $10 \%$ for the entire range of $T_{\mathrm{e}}$ and $n_{\mathrm{e}}$ covered by the photoionized nebulae (Kehrig et al. 2015).

The total extinction-corrected $\mathrm{H} \beta$ flux from the same regions is $4.44 \times 10^{-12} \mathrm{erg} \mathrm{cm}^{-2} \mathrm{~s}^{-1}$. This is equivalent to a Lyman continuum rate $\mathrm{Q}\left(\mathrm{H}^{0}\right)$ of $1.0 \times 10^{52}$ photon s $^{-1}$, using an equation similar to equation 2 but for $\mathrm{Q}\left(\mathrm{H}^{0}\right)$, which is

$$
\frac{\mathrm{Q}\left(\mathrm{H}^{0}\right)}{\text { photon s}^{-1}}=2.10 \times 10^{48} \frac{L(\mathrm{H} \beta)}{10^{36} \mathrm{erg} \mathrm{s}^{-1}},
$$

where $L(\mathrm{H} \beta)$ is the luminosity of the $\mathrm{H} \beta$ line. The above equations result in the ratio

$$
\mathrm{Q}\left(\mathrm{He}^{+}\right) / \mathrm{Q}\left(\mathrm{H}^{0}\right)=0.486 \times \frac{L(\mathrm{He} \mathrm{II} \lambda 4686)}{L(\mathrm{H} \beta)} .
$$

The luminosity of He II $\lambda 4686$ corresponds to $2.05 \%$ of the $\mathrm{H} \beta$ luminosity, which results in a value of $\mathrm{Q}\left(\mathrm{He}^{+}\right) / \mathrm{Q}\left(\mathrm{H}^{0}\right)=$ $0.0100 \pm 0.0002$.

The emission EW of $\mathrm{H} \beta$ of a starburst population is a well-known age indicator. For unresolved regions, it is one of the quantities easiest to obtain from spectroscopic data. Furthermore, it is independent of extinction as long as the ionizing cluster and the nebula are spatially coincident, or there is no differential extinction between cluster stars and nebula. However, in a resolved nebula such as the one discussed here, determination of $\operatorname{EW}(\mathrm{H} \beta)$ is non-trivial. As the ionized nebula is spatially separated from the ionizing cluster, we made the assumption that the photoionization from SSC-A is responsible for the total observed $\mathrm{H} \beta$ flux. With this assumption, we divided the observed integrated $\mathrm{H} \beta$ flux by the continuum flux in the integrated spectrum of SSCA (displayed in Fig. 4), measured close to the $\mathrm{H} \beta$ line. We obtained $\operatorname{EW}(\mathrm{H} \beta)$ under three assumptions. The first one is that the ratio is independent of extinction. In the second case, the continuum flux is corrected by $A_{\mathrm{V}}=2.3 \mathrm{mag}$, and the $\mathrm{H} \beta$ flux is corrected for the extinction determined by the Balmer decrement method. In the third case, the same $\mathrm{H} \beta$ flux correction was used, but the continuum flux was corrected by making use the minimum extinction of $A_{\mathrm{V}}=1.6 \mathrm{mag}$. The three assumptions give values of $75 \AA$, $75 \AA$, $160 \AA$, respectively.

\subsection{The number of WR stars in the mapped zone}

While fitting the $\mathrm{BB}$ with multi-Gaussians, we have looked for N (N III $\lambda \lambda 4634 / 41)$ and C (C III $\lambda \lambda 4647 / 66$ and $\mathrm{C}$ IV $\lambda 4658$ ) broad lines in addition to the broad He II $\lambda 4686$ feature. In all spectra, including that of SSC-A, only one broad component was required, which is the He II $\lambda 4686$ line. In some spectra, a nebular [Fe III] $\lambda 4658$ line is seen at the expected broad C IV $\lambda 4658$ line, where our fitting procedure 


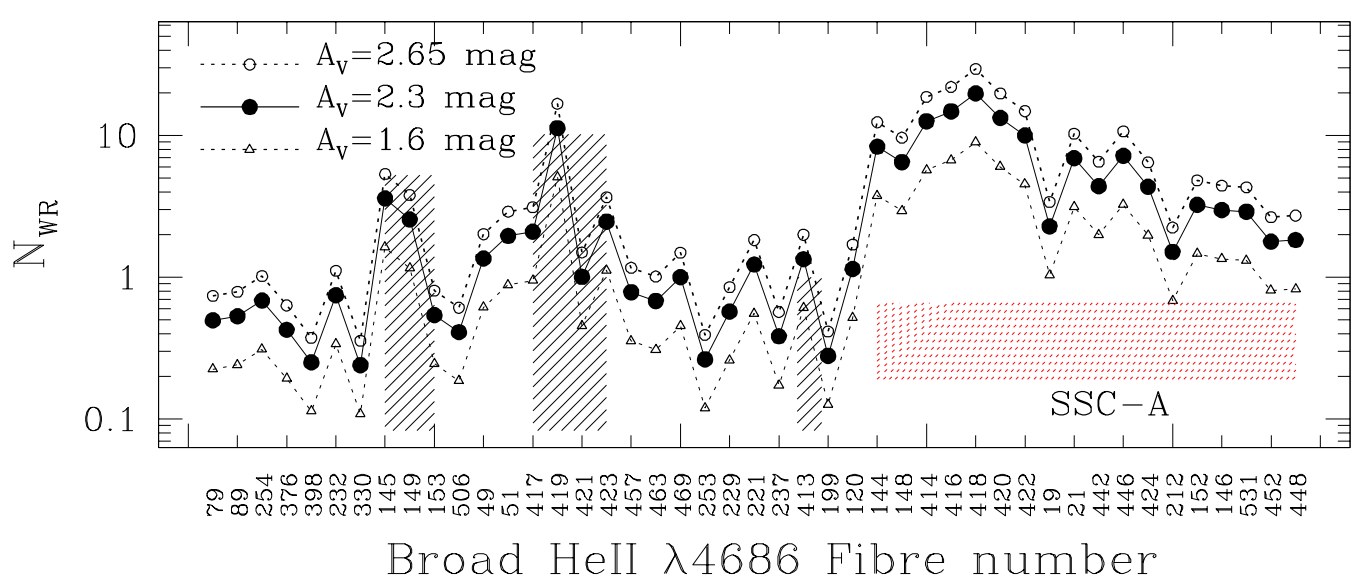

Figure 8. The number of WR stars, defined as the observed He II $\lambda 4686$ broad line luminosity divided by the typical luminosity of a WNL star $\left(1.22 \times 10^{36} \mathrm{erg} \mathrm{s}^{-1}\right)$, vs fibre number. All 50 individual fibres where we detected broad He II $\lambda 4686$ feature are shown. The numbers are plotted for three values of extinction: $A_{\mathrm{V}}=1.6 \mathrm{mag}$, the minimum along the line of sight to NGC 1569 (triangles), $A_{\mathrm{V}}=2.65 \mathrm{mag}$, the mean determined for the ionized nebula (open circles), and $A_{\mathrm{V}}=2.3 \mathrm{mag}$, optimum value for SSC-A (solid circles). Fibres belonging to SSC-A, and those that have cross-talk with fibres of SSC-A are indicated by red shaded area, and diagonally hatched area, respectively. SSC-A contains $124 \mathrm{WR}$ stars for $A_{\mathrm{V}}=2.3 \mathrm{mag}$.

would have been able to recover a broad feature even in the presence of an overlying narrow line. The absence of any of the $\mathrm{C}$ lines points to the absence of WC stars in the observed zone. Previous observations of SSC-A that had covered the red bump (C IV $\lambda \lambda 5801 / 12$ ) part of the spectrum had already indicated the absence of WC stars (González-Delgado et al. 1997), which is not unusual given that its metallicity is lower than solar (López-Sánchez \& Esteban 2010). Thus, we conclude that all our detections originated in WN stars. The relative weakness of $\mathrm{N}$ III $\lambda \lambda 4634 / 41$ suggests these are WNL stars.

We now estimate the number of WR stars in each of the fibres where the broad He II $\lambda 4686$ feature was detected in single-fibre spectra. As pointed out in $\S 3.2,18$ of these fibres belong to SSC-A, and 8 other fibres are in cross-talk with fibres belonging to SSC-A. Single-fibre detections in rest of the fibre spectra are considered tentative, as the detected broad feature could not be recovered by summing spectra of neighbouring fibres. The number was estimated by dividing the observed luminosity in the He II $\lambda 4686$ broad feature by the typical luminosity of a WNL star which is $\sim 1.22 \times 10^{36} \mathrm{erg} \mathrm{s}^{-1}$ (López-Sánchez \& Esteban 2010) for the metallicity of NGC 1569 . This value is $\sim 30 \%$ smaller than the corresponding value at the Solar metallicity (Vacca \& Conti 1992). The observed luminosities of the He II $\lambda 4686$ broad feature in each fibre need to be corrected for extinction. As discussed above, the $A_{\mathrm{V}}$ values derived at the positions of WR stars are highly unreliable, because of low nebular flux and high underlying Balmer absorptions at these locations. Larsen et al. (2011) found the colours of the resolved stellar population of SSC-A to be consistent with $A_{\mathrm{V}}=2.3 \mathrm{mag}$. This suggests that the internal extinction of the cluster stars is 0.35 mag lower than the mean value of the nebula, as expected for an attenuation law like the one proposed by Calzetti, Kinney \& Storchi-Bergmann (1994). Hence, $A_{\mathrm{V}}=2.3 \mathrm{mag}$ seems the most appropriate value to use to correct the fluxes of the He II $\lambda 4686$ broad feature from SSC-A. We hence calculate the number of WR stars using this optimum value $A_{\mathrm{V}}=2.3 \mathrm{mag}$. We also calculate the minimum and maximum number corresponding to $A_{\mathrm{V}}=1.6 \mathrm{mag}$ and $A_{\mathrm{V}}=2.65 \mathrm{mag}$, respectively.

In Fig. 8, we show the estimated number of WR stars in each fibre where the broad He II $\lambda 4686$ feature is inferred, including those fibres with tentative detections. The solid symbols joined by solid lines indicate the number using the optimum value for SSC-A of $A_{\mathrm{V}}=2.3 \mathrm{mag}$, whereas the other two lines correspond to minimum $\left(A_{\mathrm{V}}=1.6 \mathrm{mag}\right.$, triangles joined by dotted lines $)$ and mean nebular $\left(A_{\mathrm{V}}=2.65 \mathrm{mag}\right.$, circles with dotted lines) extinction. Fibres assigned to SSC-A are identified (red shaded area), as well the 8 fibres that are affected by cross-talk with SSC-A (black diagonal hatched area). With the optimum value of $A_{\mathrm{V}}=2.3 \mathrm{mag}$, all the fibres associated to SSC-A have the luminosity of at least $1 \mathrm{WNL}$ star, with one of the fibres (\#418) containing as many as 19 WNLs. This fibre belongs to the core of SSC-A. In total, we find $124 \pm 11$ WNL stars in SSC-A by adding the numbers in each one of the 18 fibres, where the quoted error assumes poissonian statistics. This number agrees with the corresponding number obtained by fitting the multi-Gaussian on the spectra summed over 19 fibres covering the SSC-A. Detected fluxes correspond to $56 \pm 7$ and $186 \pm 13$ WNL stars if we adopt $A_{\mathrm{V}}=1.6 \mathrm{mag}$ or $A_{\mathrm{V}}=2.65 \mathrm{mag}$, respectively. The uncertainty in the modeling of the extended wings of the spatial profile of the PSF of the fibres for those SSC-A spaxels where the WR bump is detected might lead to an underestimation to the total number of WR stars in SSC-A that could be of the order of a few per cent. Among the other single-fibre detections, only sources belonging to fibres \#49 and \#51 have $N_{W R}$ at least one for $A_{\mathrm{V}} \geqslant 2.3 \mathrm{mag}$. Detected fluxes in the rest of the fibres are less than the flux expected for a WNL star. As discussed in $\S 3.2$, these detections were considered as tentative due to the non-recovery of the broad component in spectra obtained by summing spectra of neighbouring fibres.

González-Delgado et al. (1997) have estimated the number of WR stars in SSC-A from their long-slit spectro- 
scopic data assuming an $A_{\mathrm{V}}=1.6 \mathrm{mag}$. Using an older distance estimate of $2.2 \mathrm{kpc}$, and luminosity of a WNL star of $\sim 1.7 \times 10^{36} \mathrm{erg} \mathrm{s}^{-1}$ from Vacca \& Conti (1992), they estimated 24 WNL stars from their spectra, which corresponds to $66 \mathrm{WNL}$ stars for the values used in our work. Our value for $A_{\mathrm{V}}=1.6 \mathrm{mag}$ is comparable with this value. On the other hand, the number of WR stars estimated based on the HST narrow band image in the F469N filter by Buckalew et al. $(2000)$ is $51 \pm 19\left(A_{\mathrm{V}}=1.6 \mathrm{mag}\right.$, distance $\left.=2.2 \mathrm{kpc}\right) \mathrm{WNL}$ stars, which corresponds to 141 WNLs for the distance and WNL luminosity used in our work. This is 2.5 times higher than our value. As noted earlier (see Fig. 4 for SSC-A), nebular lines are weak for SSC-A, and hence it is unlikely that this discrepancy comes from the contribution of the nebular lines (e.g. He II $\lambda 4686$, He I $\lambda 4713$ and [Fe III] $\lambda 4658$ ) to the inferred flux in the F469N filter. Residual error in continuum subtraction because of the strong continuum of SSC-A could be the most likely reason for the overestimation of the number of WR stars.

\section{THE IONIZATION BUDGET OF He II}

\subsection{Ionization by WR stars}

The region of NGC 1569 analyzed in this work is dominated by the SSCs A and B in continuum light (Arp \& Sandage 1985). Prada, Greeve \& McKeith (1994) inferred the presence of Red Super Giants (RSGs) in both the SSCs A and B. SSC-A is made of two components, separated by $0.2 \operatorname{arcsec}(3 \mathrm{pc})$, called A1 and A2 by De Marchi et al. (1997). González-Delgado et al. (1997) found spectral features characteristic of both the WR stars and RSGs in their ground-based long-slit spectra of SSC-A. Origlia et al. (2001) analysed HST UV spectra of SSC-A and suggested that the RSGs and WR features are orginiated in A1 and A2 components, respectively, with the age of the latter component not exceeding 5 Myr. Larsen et al. (2011) carried out photometry of the stars in the periphery of the clusters A and $\mathrm{B}$ on the HST images to obtain turn-off ages in the colourmagnitude diagram (CMD). They found RSGs in the CMD of SSC-B, but not in SSC-A. If the RSGs are restricted to the A1 component as Origlia et al. (2001) suggested this implies this component is centrally concentrated and the stars in the periphery are part of A2, the younger of the two subclusters. Based on the absence of RSGs in the CMD, they derived an upper age limit of 4.5 Myr for this component. On the other hand, they obtained an age of $16 \mathrm{Myr}$ for SSC-B. Thus, at present SSC-A is in the WR phase, when a cluster is most efficient in producing He II ionizing photons. We point out that the bright $\mathrm{H} \alpha$ emission seen at the top-right corner of the image presented in Fig. 5 is due to ionization by cluster 10 (Westmoquette et al. 2007), which coincides with WR cluster C1 of Buckalew et al. (2000). We here investigate whether SSC-A alone is capable of producing the observed luminosity of the He II $\lambda 4686$ nebular line.

The principal sources of He II ionizing photons in young stellar systems are $\mathrm{O}$ stars and their evolutionary products, such as WR stars. These stars have extended atmospheres with high mass-loss rates, hence a calculation of the rate of He II ionizing photons has to take into account the radiation transfer through these atmospheres. In recent years, such spectra are available from CMFGEN code (Pauldrach, Hoffmann \& Lennon 2001; Hillier \& Miller 1998; Smith, Norris, Crowther 2002) for O and WR stars, and from the Potsdam PoWR library (Gräfener, Koesterke \& Hamann 2002) for WR stars. We compared our observed values with publicly available Simple Stellar Population (SSP) models that have incorporated either CMFGEN or PoWR spectra in their codes. Calculations are available at discrete values of metallicity. The gas-phase oxygen abundance of NGC 1569 corresponds to $\mathrm{Z}=0.006$ for a depletion of $30 \%$ of oxygen on to dust grains and assuming $[\mathrm{O} / \mathrm{Fe}]=0.0$ (Gutkin, Charlot \& Bruzual 2016). We illustrate the results for models using $\mathrm{Z}=0.008$, which is the closest metallicity to that of NGC 1569, and comment on the metallicity dependence of the obtained results. A summary of the $\mathrm{Z}=0.008$ models we used is given in Table 1 .

The codes we used are, STARBURST99 (Leitherer et al. 1999; Leitherer, et al. 2014) with Padova (SB99/Padova; Bressan, Chiosi, Fagotto 1994) and Geneva high-mass loss (SB99/Geneva; Meynet, et al. 1994) evolutionary tracks, PopStar (PopStar/Padova; Mollá, García-Vargas \& Bressan 2009) with an updated version of the Bressan, Chiosi, Fagotto (1994) Padova tracks, two versions of GALAXEV: BC03/Padova (Bruzual \& Charlot 2003) based on tracks from Bressan, Chiosi, Fagotto (1994) and C\&B/Padova (Charlot \& Bruzual, in preparation; see Gutkin, Charlot \& Bruzual 2016; Plat et al. 2019) based on tracks from Chen et al. (2015), and the BPASS (Eldridge et al. 2017) that uses Cambridge evolutionary tracks in single (BPASS/single) and binary (BPASS/binary) mode. STARBURST99 and PopStar make use of CMFGEN code, whereas GALAXEV and BPASS use PoWR code, to model the extended atmospheres of WR stars. We downloaded the latest results from these codes from the websites of the respective codes, uniformly using Kroupa (2001) IMF between $0.15 \mathrm{M}_{\odot}$ to $100 \mathrm{M}_{\odot}$. In the case of BPASS models, the detailed calculations of ratios of different lines, including that of $\mathrm{I}(\mathrm{He}$ II $\lambda 4686) / \mathrm{I}(\mathrm{H} \beta)$, has been provided by Xiao, Stanway \& Eldridge (2018). We used the ratios corresponding to the ionization parameter of $\log U=-1.5$ and an atomic density of 1 atom $\mathrm{cm}^{-3}$. For the rest of the models, we used Eqn. 5 to convert the ratio of $\mathrm{He}^{+}$to $\mathrm{H}^{0}$ ionizing photon rates to a flux ratio $\mathrm{I}(\mathrm{He}$ II $\lambda 4686) / \mathrm{I}(\mathrm{H} \beta)$.

Results of the comparisons are presented in Fig. 9 on the left panels. Horizontal hatched areas in each panel encompass the entire range of observable quantities with account for observational errors. For I(He II $\lambda 4686) / \mathrm{I}(\mathrm{H} \beta)$ and $\mathrm{Q}\left(\mathrm{H}^{0}\right)$, errors are taken as $10 \%$, whereas for the number of WR stars, the main source of error is the uncertainty in extinction correction.

The model $\mathrm{Q}\left(\mathrm{H}^{0}\right)$ decreases monotonically with age, whereas the nebular I(He II $\lambda 4686) / \mathrm{I}(\mathrm{H} \beta)$ ratio has a welldefined peak at $\sim 4 \mathrm{Myr}$, which corresponds to the appearance of WR stars in the cluster. This ratio is independent of distance, cluster mass, extinction, the chosen nebular parameters (temperature and density), and the choice of IMF parameters, as long as the upper cut-off mass is not very much different from $100 \mathrm{M}_{\odot}$. Hence, among the three plotted quantities, the age derived from $\mathrm{I}(\mathrm{He}$ II $\lambda 4686) / \mathrm{I}(\mathrm{H} \beta)$ ratio is the most reliable. Three models that use Bressan, Chiosi, Fagotto (1994) Padova evolutionary tracks have peak values of $\mathrm{I}(\mathrm{He}$ II $\lambda 4686) / \mathrm{I}(\mathrm{H} \beta)$ higher than the observed 

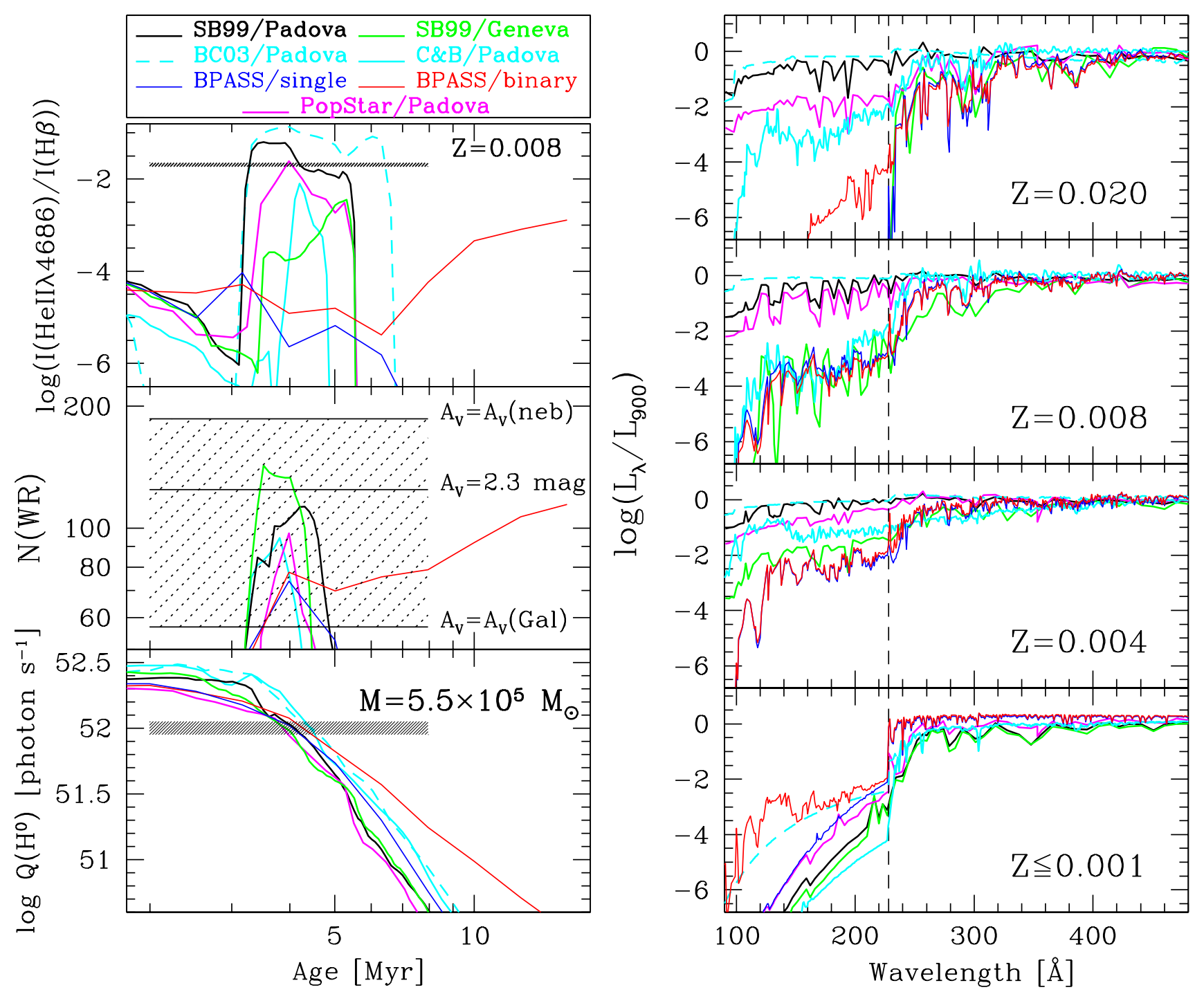

Figure 9. (Left panels) Comparison of observed quantities in NGC 1569 with Population Synthesis models. The plotted observed quantities (horizontal hatched areas) are the ratio of integrated nebular He II $\lambda 4686$ line flux to that of $\mathrm{H} \beta$ line (top-left), number of WR stars in SSC-A (middle-left) and the rate of hydrogen ionizing photons determined from the integrated $\mathrm{H} \beta$ flux (bottom-left). Results from 7 different population synthesis models are shown, which are identified in a box above the plots (see text and Table 1 for details of these models). Models that use Padova evolutionary tracks are able to reproduce the observed values for a cluster of mass $=5.5 \times 10^{5} \mathrm{M}_{\odot}$ at an age of 4 Myr. All models use Kroupa IMF at $Z=0.008$, which is the metallicity closest to that of NGC 1569 available in all the codes. (right panels) Spectral shape near the $\mathrm{He}^{+}$ionizing edge $(228 \AA)$ at the 4 indicated metallicities during the WR phase. Spectra are shown normalised to their flux at $900 \AA$ wavelength. All spectra show a jump blueward of $228 \AA$ at the lowest plotted metallicity. However, at higher metallicities the spectral shapes blueward of $228 \AA$ for the plotted models do not coincide, with the spectra being harder in models incorporating Padova tracks as compared to those obtained using other tracks.

value in NGC 1569 (see Tab. 1). The ratio is marginally smaller for model that makes use of the results from the new Padova tracks (Chen et al. 2015), whereas in the other three models this ratio is much lower. All the models reproduce the observationally estimated $\mathrm{Q}\left(\mathrm{H}^{0}\right)$ for a cluster of mass $(5.5 \pm 0.5) \times 10^{5} \mathrm{M}_{\odot}$ in its WR phase. The observed $\mathrm{EW}(\mathrm{H} \beta)$ is also consistent with the value expected during the WR phase for single star evolutionary models (see column 7 in Tab. 1). The number of WR stars predicted in all models agrees with the best estimation of the observed value to within $30 \%$ for the mass inferred above. The WR phase in single star evolutionary models lasts between $\sim 3.5-$ 5 Myr. The limits for star cluster age are determined from the condition that the model predicted number of WR stars for the above inferred mass exceeds the lower limit for the observationally inferred number of WR stars in SSC-A. This age range, as well as the age at which $\mathrm{I}(\mathrm{He} \mathrm{II} \lambda 4686) / \mathrm{I}(\mathrm{H} \beta)$ 
Table 1. Comparison of Population Synthesis model results for $\mathrm{Z}=0.008$ with observations of SSC-A

\begin{tabular}{|c|c|c|c|c|c|c|c|c|}
\hline \multirow{2}{*}{$\begin{array}{l}\text { SSP code ID } \\
\text { (1) }\end{array}$} & \multirow{2}{*}{$\begin{array}{l}\text { WR model }+ \text { atmosphere } \\
\text { (2) }\end{array}$} & \multicolumn{2}{|c|}{$\mathrm{I}(\mathrm{He} \mathrm{II}) / \mathrm{H} \beta$} & \multicolumn{2}{|c|}{ WR phase } & \multirow{2}{*}{$\begin{array}{c}\mathrm{EW}(\mathrm{H} \beta) \\
\AA \\
(7)\end{array}$} & \multirow{2}{*}{$\begin{array}{l}\mathrm{Q}(\mathrm{H} 0) \\
\log \\
\mathrm{ph} \mathrm{s}^{-1} \\
(8)\end{array}$} & \multirow{2}{*}{$\begin{array}{l}\text { Comments } \\
\text { (9) }\end{array}$} \\
\hline & & $\begin{array}{c}\text { age } \\
\text { Myr } \\
(3)\end{array}$ & $\begin{array}{c}\text { peak } \\
\text { log } \\
(4)\end{array}$ & $\begin{array}{l}\text { age } \\
\text { Myr } \\
(5)\end{array}$ & $\begin{array}{c}N_{\mathrm{WR}} \\
(6)\end{array}$ & & & \\
\hline SB99/Padova & Padova1994+CMFGEN & 3.5 & -1.20 & $3.3-4.9$ & 123 & $278-88$ & 52.03 & Good fit \\
\hline SB99/Geneva & Geneva1994+CMFGEN & 5.3 & -2.45 & $3.2-4.4$ & 155 & $157-85$ & 51.98 & $\mathrm{I}(\mathrm{He} \mathrm{II}) / \mathrm{I}(\mathrm{H} \beta)$ too low \\
\hline PopStar/Padova & Padova1994+CMFGEN & 4.0 & -1.61 & $3.5-4.5$ & 106 & $165-84$ & 51.95 & Good fit \\
\hline BC03/Padova & Padova1994+PoWR & 4.0 & -1.03 & - & - & - & 52.13 & Good fit \\
\hline C\&B/Padova & Padova2015+PoWR & 4.2 & -2.10 & $3.3-4.2$ & 89 & - & 52.10 & Marginal fit \\
\hline BPASS/single & Cambridge+PoWR & 3.2 & -4.03 & $4.0-5.0$ & 80 & $180-110$ & 52.02 & $\mathrm{I}(\mathrm{He} \mathrm{II}) / \mathrm{I}(\mathrm{H} \beta)$ too low \\
\hline BPASS/binary & Cambridge + PoWR & 16 & -2.89 & $4.0-16$ & 124 & $195-19$ & 52.08 & $\mathrm{I}(\mathrm{He} \mathrm{II}) / \mathrm{I}(\mathrm{H} \beta)$ too low \\
\hline SSC-A & Observed or inferred & & .69 & $4.0 \pm 0.5$ & $\begin{array}{c}124 \\
56-186\end{array}$ & $\begin{array}{c}75 \\
160\end{array}$ & 52.00 & $(5.5 \pm 0.5) \times 10^{5} \mathrm{M}_{\odot}$ \\
\hline
\end{tabular}

(1)-(2): Model name, evolutionary tracks and WR atmospheric models used (Padova1994=Bressan, Chiosi, Fagotto 1994; Geneva1994=Meynet, et al. 1994; Padova2015=Chen et al. 2015). Last row contains observationally inferred quantities. The tracks used in PopStar is a modified version of Padova1994 tracks as described in Mollá, García-Vargas \& Bressan (2009); (3) age at which nebular I(He II $\lambda 4686) / \mathrm{I}(\mathrm{H} \beta)$ ratio is maximum; (4) maximum value of $\mathrm{I}(\mathrm{He}$ II $\lambda 4686) / \mathrm{I}(\mathrm{H} \beta)$ in the model in log units; (5) The age interval over which the model has more than the observationally estimated minimum number of 56 WR stars, for the determined mass.

The last row contains the age range over which models using Padova tracks reproduce the observed I(He II $\lambda 4686) / \mathrm{I}(\mathrm{H} \beta)$; (6) The maximum number of WR stars in the model in the WR phase. Last row contains the best estimation and the possible range depending on the assumption on extinction; $(7)$ range of $\mathrm{EW}(\mathrm{H} \beta)$ for the age range in column 5 . $\mathrm{EW}(\mathrm{H} \beta)$ decreases monotonically with age.

Observed range in last row corresponds to the minimum and maximum values based on different assumptions on differential extinction (see text for details); (8) model $\log \left(\mathrm{Q}\left(\mathrm{H}^{0}\right)\right)$ at the most likely age $(4 \mathrm{Myr})$ and mass $\left(5.5 \times 10^{5} \mathrm{M}_{\odot}\right)$; $(9)$ comments on the comparison between the observations and model. Last row contains the best determined mass and error on it, which takes into account the error on the determined age.

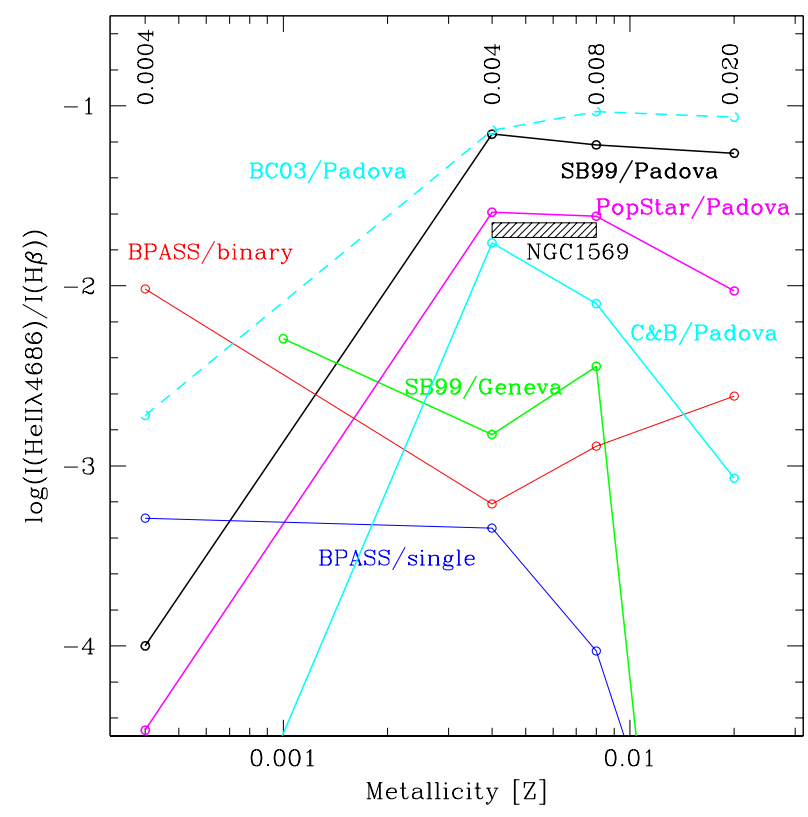

Figure 10. Maximum values of the nebular I(He II $\lambda 4686) / \mathrm{I}(\mathrm{H} \beta)$ ratio (points joined by lines) reached as a function of the abundance for the SSP models indicated close to each curve. The observed value for NGC1569 is indicated, where the range in metallicity takes into account $10-30 \%$ depletion of oxygen into dust grains (Gutkin, Charlot \& Bruzual 2016). The observed values are in agreement with the highest values of model $\mathrm{I}(\mathrm{He}$ II $\lambda 4686) / \mathrm{I}(\mathrm{H} \beta)$ ratio, that happens between $\mathrm{Z}=0.004$ and $\mathrm{Z}=0.008$ for new (C\&B/Padova) as well as old Padova (SB99/Padova, PopStar/Padova) models. ratio reaches its peak value, are given in Table 1 , in columns 5 and 3 , respectively.

It is interesting to note that the SB99/Padova and SB99/Geneva models use the same atmospheric models in the WR phase, but Geneva evolutionary tracks have lower $\mathrm{I}(\mathrm{He}$ II $\lambda 4686) / \mathrm{I}(\mathrm{H} \beta)$ in spite of having $\sim 25 \%$ more number of WR stars. This implies that the low values of $\mathrm{I}(\mathrm{He}$ II $\lambda 4686) / \mathrm{I}(\mathrm{H} \beta)$ in SB99/Geneva models are not due to lack of WR stars, but due to the different surface parameter values in these models as compared to those in the Padova tracks. Both the single and binary BPASS models predict systematically a smaller number of WR stars, and a lower peak value of $\mathrm{I}(\mathrm{He}$ II $\lambda 4686) / \mathrm{I}(\mathrm{H} \beta)$ at the $3-5 \mathrm{Myr}$ age. In binary models, peak values of these two parameters are reached at later ages $(\sim 16 \mathrm{Myr})$ when $\mathrm{Q}\left(\mathrm{H}^{0}\right)$ falls by a factor of 30 with respect to its values at the WR phase in single star evolutionary models. The observed $\mathrm{EW}$ of $\mathrm{H} \beta$ is not in favour of this advanced age.

In the right panel of Fig. 9, we plot the typical emergent spectum around the $\mathrm{He}^{+}$ionization edge $(228 \AA$; the dashed vertical line) during the WR phase (4 Myr for all models except BPASS where it is $3.2 \mathrm{Myr}$ ) for a range of metallicities. All spectra are normalized to their fluxes at $\lambda=900 \AA$. The normalisation wavelength is specifically chosen to be slightly blueward of the $\mathrm{H}^{0}$ ionization edge (912 $\AA$ ), so that the shape of the plotted spectra blueward of $228 \AA$ is an indicator of the $\mathrm{I}(\mathrm{He} \mathrm{II} \lambda 4686) / \mathrm{I}(\mathrm{H} \beta)$ ratio. The spectra in the four models incorporating the Padova tracks (SB99/Padova, PopStar, BC03 and C\&B) exhibit more $\mathrm{He}^{+}$ ionizing photons blueward of $228 \AA$ for metallicities $\mathrm{Z} \geqslant 0.004$ than those in the SB99/Geneva and BPASS models. At these metallicities, the emergent spectrum using the new Padova tracks (C\&B/Padova) is softer than that using the older Padova tracks, but it is still harder than that obtained in 
SB99/Geneva and BPASS models. On the other hand, the spectral behaviour is similar in all the seven plotted models at low metallicities $(\mathrm{Z} \leqslant 0.001)$, all models predicting a downward jump at $228 \AA$. The plot illustrates that the atmospheric parameters (effective temperature and bolometric luminosity) of WR stars in Padova tracks are particularly able to account for the observed ratio of I(He II $\lambda 4686) / \mathrm{I}(\mathrm{H} \beta)$ at metallicities $\mathrm{Z} \geqslant 0.004$.

In the above analysis we have compared the values of NGC 1569 with $Z=0.008$ metallicity models. For the observed gas-phase abundance of oxygen, the metallicity $\mathrm{Z}$ can be as low as $\mathrm{Z}=0.004$ if less than $10 \%$ of oxygen is depleted on to dust grains (Gutkin, Charlot \& Bruzual 2016). Given that the hardness of the spectrum blueward of the $\mathrm{He}^{+}$ionization edge depends on metallicity, we now examine the behaviour of $\mathrm{I}(\mathrm{He}$ II $\lambda 4686) / \mathrm{I}(\mathrm{H} \beta)$ ratio with metallicity during the WR phase. This is illustrated in Fig. 10 for all the models discussed in this work. The observed ratio in NGC 1569 is well reproduced for a metallicity range of $\mathrm{Z}=0.004-0.008$ in all SSPs that use Padova tracks, independent of whether CMFGEN or PoWR models are used to represent the atmospheres of WR stars. In comparison, SSP models using Geneva tracks and BPASS models predict more than an order of magnitude lower values.

It can be seen in Fig. 10 that the $\mathrm{I}(\mathrm{He}$ II $\lambda 4686) / \mathrm{I}(\mathrm{H} \beta)$ ratio decreases sharply at $\mathrm{Z}<0.004$ in models using Padova tracks. The maximum $\mathrm{I}(\mathrm{He} \mathrm{II} \lambda 4686) / \mathrm{I}(\mathrm{H} \beta)$ ratio at the lowest plotted metallicity $(\mathrm{Z}=0.0004)$ corresponds to the BPASS/binary model. However, the ratio just about reaches 0.01 . The observed ratio in metal-poor galaxies is often higher that these predicted values (see Shirazi \& Brinchmann 2012). In fact, observed data show a gradual tendency for the nebular I(He II $\lambda 4686) / \mathrm{I}(\mathrm{H} \beta)$ ratio to increase with decreasing metallicity reaching values as high as $\sim 0.06$ in the most metal-poor galaxies known (Schaerer, Fragos \& Izotov 2019). This incapability of the SSP models to reproduce the observed $\mathrm{I}(\mathrm{He}$ II $\lambda 4686) / \mathrm{I}(\mathrm{H} \beta)$ ratio at low-metallicities $(\mathrm{Z}<0.004)$ is often referred to as the $\mathrm{He}^{+}$ionization budget problem. The problem starts arising just below the metallicity of NGC 1569. At these low metallicities, the problem is aggravated due to the small number of WR detections. For example, I Zw 18, one of the most metal-poor galaxies, emits as much $\mathrm{He}^{+}$ionizing photons as SSC-A in NGC 1569, but it has at the most $9 \mathrm{WR}$ stars detected (Kehrig et al. 2015) as compared to the $124 \mathrm{WR}$ stars in SSC-A. A comprehensive analysis of this problem was carried out recently by Plat et al. (2019). They used the same C\&B/Padova model that we have used here, and calculated the I(He II $\lambda 4686) / \mathrm{I}(\mathrm{H} \beta)$ ratio for a variety of additional input physics not explored in this study. They find that the conditions most favourable to produce $\mathrm{I}(\mathrm{He}$ II $\lambda 4686) / \mathrm{I}(\mathrm{H} \beta)>0.01$ at low metallicities include: (1) the presence of stars significantly more massive than $100 \mathrm{M}_{\odot},(2)$ extremely high ionization parameter, $\log (U)>-1,(3)$ the presence of interacting binaries that produce X-rays, (4) ionization of $\mathrm{He}^{+}$by radiative shocks, or (5) when analysing intergrated spectra of distant galaxies, ionization of $\mathrm{He}^{+}$by an active galactic nucleus. It is likely that more than one of these conditions are met in some of the metal-poor galaxies.

In summary, the observed number of $\mathrm{Q}\left(\mathrm{H}^{0}\right)$ from nebula surrounding SSC-A and $N_{\mathrm{WR}}$ in SSC-A are consistent with each other for all models for a cluster mass of
$(5.5 \pm 0.5) \times 10^{5} \mathrm{M}_{\odot}$ at $\sim 4.0 \pm 0.5 \mathrm{Myr}$ age. The observed nebular $\mathrm{I}(\mathrm{He} \mathrm{II} \lambda 4686) / \mathrm{I}(\mathrm{H} \beta)$ ratio is in the range of predicted values during the WR phase in models that use Padova evolutionary tracks. The inferred age for SSC-A is in agreement with the age of 4.5 Myr determined by Larsen et al. (2011) using the CMD of stars in the periphery of SSC-A. The inferred mass is $\sim 15 \%$ lower as compared to the photometric mass of $6.3 \times 10^{5} \mathrm{M}_{\odot}$ determined by Larsen et al. (2011), after scaling their mass to the distance of $3.1 \mathrm{Mpc}$ used in this work. Slightly larger mass for SSC-A in Larsen et al. (2011) is expected given that the SSC-A has two populations, a centrally concentrated population (A1) containing RSGs (older than $7 \mathrm{Myr}$ ) and a slightly extended component (A2) containing WR and O stars. Our measured mass is based on the ionizing flux and hence corresponds to the mass of the component A2. On the other hand, the photometric mass derived by Larsen et al. (2011) is based on integrated photometry of stellar light, and hence it includes the mass of both the components. On the other hand, the mass derived by us is $\sim 25 \%$ higher than the dynamical mass of $4.1 \times 10^{5} \mathrm{M}_{\odot}$ obtained by Ho \& Filippenko (1996) using spectral lines originating in cool supergiants. Ho \& Filippenko (1996) commented that the velocity dispersion obtained from cool supergiants in the integrated spectrum could underestimate the mass by as much as a factor of 2 . Furthermore, the cool supergiants belong to the older of the two populations. The derived dynamical mass is expected to be the total of the two populations if they are dynamically mixed. The presence of hot massive stars, but not cool supergiants, in the periphery of SSC-A argues against such a mixing (Larsen et al. 2011). Measurements of velocity dispersion using spectral lines sensitive to hot stars (e.g. He absorption lines which are prominent in the spectrum of SSC-A) could help to address this issue. MEGARA has the capability of obtaining velocity dispersion in its high resolution mode, providing a possibility of addressing this issue in the near future.

\subsection{Morphology of the He II $\lambda 4686$ nebula and the location of SSC-A}

We found that the entire observed He II $\lambda 4686$ emission can be understood in terms of the ionization from WR stars in SSC-A. However, the observed He II $\lambda 4686$ emission is not coincident with the location of the ionizing cluster. Instead, the most intense part of the ionized nebula both in the Balmer and in the He II $\lambda 4686$ lines lies $\sim 40$ pc to the south-west of the cluster. The zone of intense emission is part of a semicircular arc of $150 \mathrm{pc}$ diameter, with its centre $\sim 40 \mathrm{pc}$ to the east of SSC-A. Because of this off-centring, the distance of ionizing source to different parts of the emitting arc is different, causing the surface brightness of the emission to decrease along the arc as its distance increases from SSC-A. The emission is weak inside the semi-circular arc (see Section 3). The observed morphology resembles the structure of a classical wind-driven bubble. If this is the case, the hot shocked gas that fills the bubble should be a source of the X-ray emission (Weaver et al. 1977; Chu \& Mac Low 1990; Silich, Tenorio-Tagle \& Añorve-Zeferino 2005). Soft X-rays are indeed detected in NGC 1569, whose morphology is discussed below. 


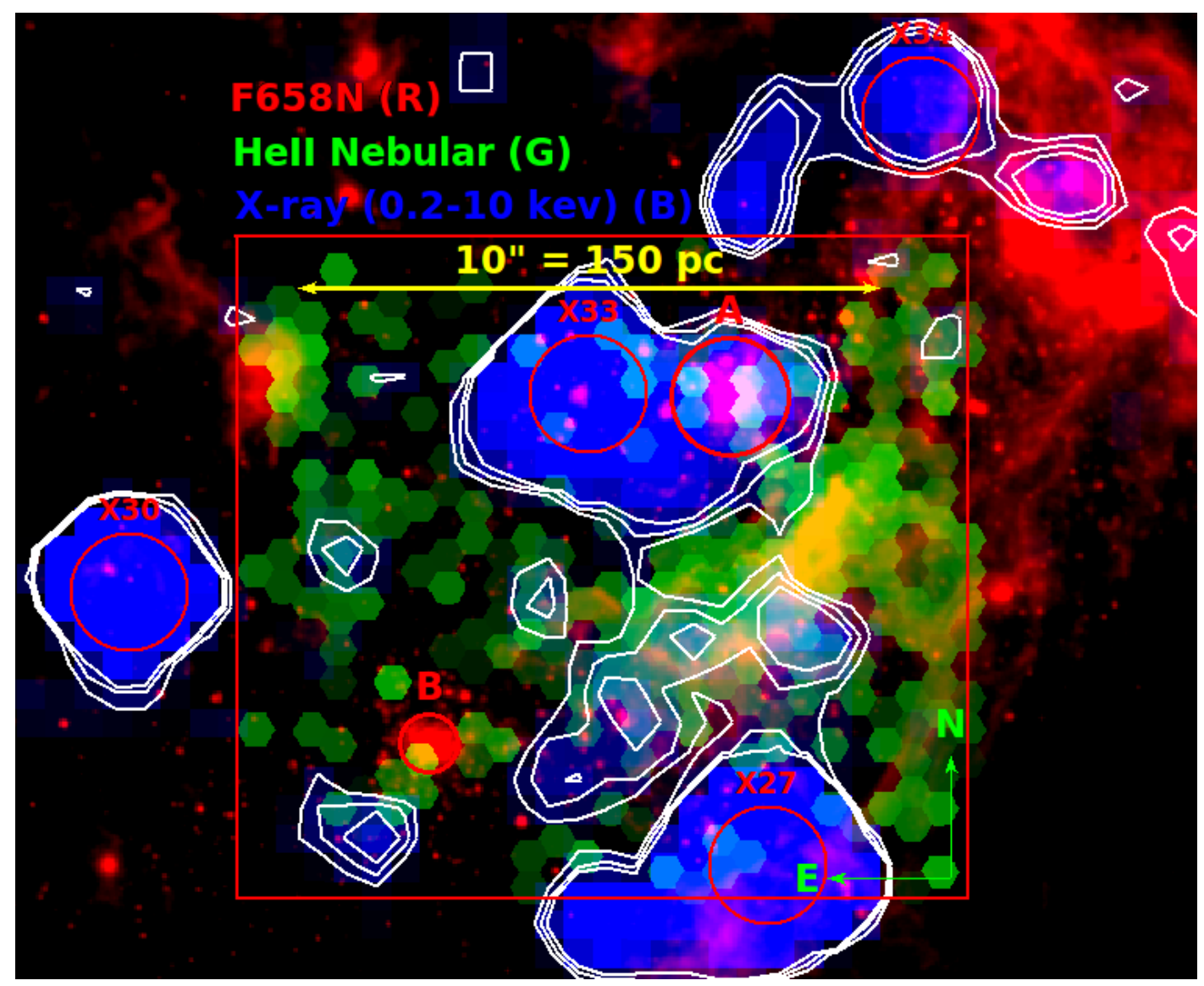

Figure 11. X-ray emission map from the Chandra/ACIS image $(0.2-10 \mathrm{keV}$ band) from Sánchez-Cruces et al. (2015) in blue is superposed on the He II nebular map in green and F658N (H $\alpha+$ continuum) in red. The contours show the X-ray emission at 3 levels $(3 \sigma$, $3.5 \sigma$ and $4 \sigma$ above the background) to help see the position of the faint X-ray diffuse emission with respect to the He II $\lambda 4686$ nebular emission. Most of the X-ray emission within the MEGARA FoV (red square) originates in two hard X-ray point sources (X27 and X33), which are marked by red circles. The diffuse X-ray emission coincides with part of the He II $\lambda 4686$ nebular emission. However, there is no X-ray emission at the brightest zone of the nebular He II $\lambda 4686$ emission.

\subsection{X-ray morphology}

The combined effect of stellar winds from massive stars in cluster A, including those from the multiple WR stars, is to form a global star cluster wind able to expel the inserted and the residual gas away into the interstellar medium (ISM). The interaction of the combined star cluster wind with the ISM leads to a strong shock which sweeps up the ambient gas into a thin shell, while the cluster wind is thermalised at a reverse shock. The shell of swept-up matter cools rapidly, if the ambient gas density is not too small, to be then completely or partially photoionized by the energetic photons escaping the cluster (Castor, McCray \& Weaver 1975; MartínezGonzález, Silich \& Tenorio-Tagle 2014).

Both soft $(0.2-2 \mathrm{keV})$ and hard $(2-10 \mathrm{keV})$ X-ray emission have been detected from the central zone of NGC 1569 (Martin et al. 2002; Sánchez-Cruces et al. 2015). The soft Xray emission is diffuse, whereas the hard X-rays principally come from point sources. We plot the X-ray map (blue) superposed on the He II nebular map (green) in Fig. 11. The F658N image ( $\mathrm{H} \alpha+$ continuum emission) is shown in red for positional reference. The observed FoV contains two hard Xray emitting point sources catalogued by Sánchez-Cruces et al. (2015): source 27 (identified as X27) is associated to an
X-ray binary, and source 33 (denoted as X33) as originating in the cluster 29 of Hunter et al. (2000) (see our Fig. 3).

Most of the soft X-ray emission comes from diffuse regions coincident with parts of the He II nebula. However, the brightest part of the He II nebula does not show any Xray emission. As illustrated by Sánchez-Cruces et al. (2015), there is faint emission from the intervening zone between the SSC-A and the ionized nebula. This emission is likely associated with the hot shocked winds.

$\mathrm{X}$-ray photons can also produce the He II emission. We used the factor $q=\mathrm{Q}\left(\mathrm{He}^{+}\right) / L_{X}=2 \times 10^{10}{\text { photon } \mathrm{erg}^{-1}}^{-1}$ defined by Schaerer, Fragos \& Izotov (2019) to estimate the $\mathrm{Q}\left(\mathrm{He}^{+}\right)$using the observed X-ray luminosity from the two $\mathrm{X}$-ray sources in our FoV. The two sources combined contribute $\sim 1.7 \times 10^{48}$ photon $^{-1}$, which is less than $2 \%$ of the observed ionization requirement. Hence, we are justified in neglecting any contribution to ionization from X-ray photons.

\section{CONCLUSIONS}

Using the recently available integral field spectrograph MEGARA at the 10.4-m GTC, we detect extended 
He II $\lambda 4686$ nebula in the central starburst zone of the nearby dwarf galaxy NGC 1569. The nebula extends along a semicircular arc of $\sim 150 \mathrm{pc}(10$ arcsec $)$ diameter and $\sim 40 \mathrm{pc}$ width, with the zone of the brightest He II $\lambda 4686$ nebular emission lying at $\sim 40 \mathrm{pc}(2.5 \mathrm{arcsec})$ south-west of the massive young cluster SSC-A. The spectral data also show broad He II $\lambda 4686$ emission from 18 individual fibres belonging to the SSC-A. None of the other sources that are well separated from SSC-A, where WR detection have been reported using HST imaging data by Buckalew et al. (2000), show broad He II $\lambda 4686$ emission. We find that these sources are stars and clusters immersed in the He II $\lambda 4686$ nebula. We use the $\mathrm{H} \beta$ and $\mathrm{H} \gamma$ nebular lines in the same spectra to map the extinction in the observed zone. The minimum extinction we obtain is consistent with the $A_{\mathrm{V}}=1.6 \mathrm{mag}$ from the Milky Way along the line of sight to NGC 1569. The mean value for all fibres where we could reliably measure $A_{\mathrm{V}}$ is $A_{\mathrm{V}}=2.65 \mathrm{mag}$. The low surface brightness of the nebular flux in and around SSC-A prevented us from obtaining nebular extinction towards this cluster. Using $A_{\mathrm{V}}=2.3 \mathrm{mag}$, the value used by Larsen et al. (2011) to interpret the colours of the resolved stellar population in SSC-A, we estimate $124 \pm 11 \mathrm{WR}$ stars of type WNL in SSC-A. We derive hydrogen and $\mathrm{He}^{+}$ionizing rates of $1 \times 10^{52}$ photon $\mathrm{s}^{-1}$ and $1 \times 10^{50}$ photon $^{-1}$, respectively. These observed quantities are in agreement with the expectations from single stellar population models at $4.0 \pm 0.5 \mathrm{Myr}$ for a cluster of mass $(5.5 \pm 0.5) \times 10^{5} \mathrm{M}_{\odot}$. The derived age is consistent with the turn-off ages determined by Larsen et al. (2011) from resolved stellar populations in the periphery of this cluster. A careful comparison of the most commonly used population synthesis models, we find that the predicted values of nebular I(He II $\lambda 4686) / \mathrm{I}(\mathrm{H} \beta)$ in models that make use of Padova evolutionary tracks are in the range of observed value of 0.02 for metallicities $\mathrm{Z} \geqslant 0.004$. Thus, at $12+\log (\mathrm{O} / \mathrm{H})=8.19(\mathrm{Z} \sim 0.006)$, which is the measured value for NGC 1569, the WR stars from the cluster are able to explain the origin of the He II $\lambda 4686$ nebula.

\section{ACKNOWLEDGEMENTS}

It is a pleasure to thank the anonymous referee for thoughtful comments that lead to a significant improvement of the manuscript. This publication is based on data obtained with the MEGARA instrument at the Gran Telescopio Canarias, installed in the Spanish Observatorio del Roque de los Muchachos, in the island of La Palma. MEGARA has been built by a Consortium led by the Universidad Complutense de Madrid (Spain) and that also includes the Instituto de Astrofísica, Óptica y Electrónica (Mexico), Instituto de Astrofísica de Andalucía (CSIC, Spain) and the Universidad Politécnica de Madrid (Spain). MEGARA is funded by the Consortium institutions, GRANTECAN S.A. and European Regional Development Funds (ERDF), through Programa Operativo Canarias FEDER 2014-2020. We thank CONACyT for the research grants CB-A1-S25070 (YDM), CB-A1-S-22784 (DRG), and CB-A1-S-28458 (SS). VMAGG is funded by UNAM DGAPA PAPIIT project number IA100318. Authors acknowledge financial support from the Spanish MINECO under grant numbers AYA2016-79724-C4-4-P, AYA2016-75808-R, AYA201790589-REOT and RTI2018-096188-B-I00.

\section{DATA AVAILABILITY}

The fluxes of principal emission lines used in this work are available in the article and in its online supplementary material. The reduced fits files on which these data are based will be shared on reasonable request to the first author.

\section{REFERENCES}

Arp, H., \& Sandage, A. 1985, AJ, 90, 1163

Brinchmann, J., Kunth, D. \& Durret, F. 2008, A\&A, 485, 657

Bressan A., Chiosi C., Fagotto F., 1994, ApJS, 94, 63

Bressan A., Granato G. L., Silva L. 1998, A\&A, 332, 135

Bruzual, A. G., Charlot, S., 1993, ApJ, 405, 538

Bruzual, A. G., Charlot, S., 2003, MNRAS, 344, 1000

Buckalew, B. A., Dufour, R. J., Shopbell, P. L. \& Walter, D. K. 2000, AJ, 120, 2402

Burstein, D. \& Heiles, C. 1984, ApJS, 54, 33

Calzetti D., Kinney A. L., Storchi-Bergmann T., 1994, ApJ, 429, 582

Cardelli, J. A., Clayton, G. C. \& Mathis, J. S. 1989, ApJ, 345, 245

Carrasco E., et al., 2018, SPIE, 1070216, SPIE10702

Castor J., McCray R., Weaver R., 1975, ApJL, 200, L107

Chen, Y., Bressan, A., Girardi, L., et al. 2015, MNRAS 452, 1068

Chu Y.-H., Mac Low M.-M., 1990, ApJ, 365, 510

De Marchi, G., Clampin, M., Greggio, L., Leitherer, C., Nota, A., \& Tosi, M. 1997, ApJ, 479, L27

Devost, D., Roy, J.-R. \& Drissen, L. 1997, ApJ, 482, 765

Dopita, M. A. \& Sutherland, R. S. 1996, ApJS, 102, 161

Eldridge J. J., Tout C. A., 2004, MNRAS, 353, 87

Eldridge, J. J., Stanway, E. R., Xiao, L. et al. 2017, PASA, 34, e058

Fragos T., et al., 2013, ApJ, 764, 41

Garnett, D. R., Kennicutt, R. C., Chu, Y.-H. \& Skillman, E.D. 1991, ApJ, 373, 458

Gil de Paz A., et al., 2018, SPIE, 1070217, SPIE10702

Gil de Paz A., et al., 2020 (submitted to A\&A)

Gómez-González, V.M.A., et al. 2020, MNRAS, 493, 3879

González-Delgado, R. M., Leitherer, C., Heckman, T. \& Cerviño, M. 1997, ApJ, 483, 705

Gräfener, G., Koesterke, L. \& Hamann, W.-R. 2002, A\&A, 387, 244

Grocholski, A. J., Aloisi, A., van Der Marel, R. P., et al. 2008, ApJ, 686, L79

Grocholski, A. J., van der Marel R. P., Aloisi A., Annibali F., Greggio L., Tosi M., 2012, AJ, 143, 117

Gutkin J., Charlot S. \& Bruzual, G. 2016, MNRAS, 462, 1757

Hillier, D.J. \& Miller, D.L. 1998, 496, 4007

Hunter, D. A., Hawley, W. \& Gallagher, J. S. 1993, AJ, 106, 1797

Hunter, D. A., O'Connell, R. W., Gallagher, J. S. \& SmeckerHane, T. A. 2000, AJ, 120, 2383

Ho, L. C. \& Filippenko, A. V. 1996, ApJ, 446, L83

Hodge, P. W. 1974, ApJ, 191, L21

Israel, F.P. 1988, A\&A, 194, 24

Kehrig, C., Oey, M.S., Crowther, P.A. et al. 2011, A\&A, 526, L128

Kehrig, C., Vílchez, J. M., Pérez-Montero, E. et al. 2015, ApJ, 80, L28

Kehrig, C., Vílchez, J. M., Guerrero, M. A. et al. 2018, MNRAS, 480, 1081

Kobulnicky, H. A., Skillman, E. D. 1997, ApJ, 489, 636

Kojima T., et al., 2020, arXiv, arXiv:2006.03831

Kroupa P., 2001, MNRAS, 322, 231

Larsen, S. S., Origlia, L., Brodie, J. \& Gallagher, J. S. 2008, MNRAS, 383, 263 
Larsen, S. S., de Mink, S. E., Eldridge, J. J. el al. 2011, A\&A, 532,147

Leitherer, C., Schaerer, D., Goldader, J. D., González-Delgado, R. M., Robert, C. et al. 1999, ApJ, 123, 3

Leitherer C., Ekström S., Meynet G., Schaerer D., Agienko K. B., Levesque E. M., 2014, ApJS, 212, 14

López-Sánchez Á. R., Esteban C., 2010, A\&A, 516, A104

Maeder, A. \& Meynet, G. 1989, A\&A, 210, 155

Martin, C. L., Kobulniky, H. A. \& Heckman, T. M. 2002, ApJ, 574,663

Martínez-González S., Silich S., Tenorio-Tagle G., 2014, ApJ, 785, 164

Mayya, Y. D. \& Prabhu, T. P. 1996, AJ, 111, 1252

Meynet G., Maeder A., Schaller G., Schaerer D., Charbonnel C., 1994, A\&AS, 103, 97

Meynet G., \& Maeder A., 2005, A\&A, 429, 581

Mollá, M., García-Vargas, M. L., Bressan, A., 2009, MNRAS, 398, 451

Origlia, L., Leitherer, C., Aloisi, A., Greggio, L. \& Tosi, M. 2001, AJ, 122,815

Osterbrock, D. E. \& Ferland, G. J. 2006, in Astrophysics of Gaseous Nebulae and Active Galactic Nuclei (CA: University Science Books)

Ott, T. 2012, QFitsView: FITS file viewer, ascl:1210.019

Pauldrach, A. W. A., Hoffmann, T.L. \& \& Lennon, M. 2001, A\&A, 375, 161

Pascual, S., Cardiel, N., Picazo-Sanchez, P., et al. 2018, guaixucm/megaradrp: v0.8, Zenodo, doi:10.5281/zenodo.2206856

Plat, A., Charlot, S., Bruzual, G., Feltre, A., Vidal-García, A. et al. 2019, MNRAS, 490, 978

Prada, F., Greve, A., \& McKeith, C. D. 1994, A\&A, 288, 396

Press, W.H., Teukolsky, S.A., Vetterling, W.T. \& Flannery, B.P. 1992, Numerical Recipes in C (Second Edition), Cambridge University Press

Sánchez-Cruces, M., Rosado, M., Rodríguez-González, A. \& Reyes-Iturbide, J. 2015, ApJ, 799, 231

Schaerer, D. (1996) ApJ, 467, L17

Schaerer D., Fragos T., Izotov Y. I., 2019, A\&A, 622, L10

Schaller, G., Schaerer, G. Meynet, G. \& Maeder, A. 1992, A\&AS, 96, 269

Schlafly, E. \& Finkbeiner, D. P. 2011, ApJ, 737, 103

Schlegel, D. J., Finkbeiner, D. P. \& Davis, M. 1998, ApJ, 500, 525

Schmutz W., Leitherer C., Gruenwald R., 1992, PASP, 104, 1164

Shirazi, M. \& Brinchmann, J. (2012) MNRAS, 421, 1043

Silich S., Tenorio-Tagle G., Añorve-Zeferino G. A., 2005, ApJ, 635,1116

Smith L., Norris R., \& Crowther P., 2002, MNRAS, 337, 1309

Taylor C. L., Hüttemeister S., Klein U., Greve A., 1999, A\&A, 349,424

Tresse, L., Maddox, S., Loveday, J. \& Singleton, C. 1999, MNRAS, 310, 262

Vacca, W. D. \& Conti, P. S. 1992, ApJ, 401, 543

Waller, W. H. 1991, ApJ, 370, 144

Weaver, R., McCray, R., Castor, J., Shapiro, P. \& Moore, R. 1977, ApJ, 218, 377

Westmoquette, M. S., Exter, K. M., Smith, L. J. \& Gallagher, J. S. 2007, MNRAS, 381, 894

Westmoquette, M. S., Smith, L. J. \& Gallagher, J. S. 2008, MNRAS, 383, 864

Xiao, L. et al. Stanway, E. R., Eldridge, J. J., 2018, MNRAS, 477, 904

\section{APPENDIX A: MEASURED DATA IN INDIVIDUAL FIBRE SPECTRUM}

In Table A1, we give the measured and calculated values for all the lines used in this work for 50 illustrative fibre spectra. The first 25 rows contain data for fibres arranged as a decreasing function of $\mathrm{H} \beta$ flux (column 6), whereas the remaining 25 rows show data for fibres where we detected WR features. These data are arranged in the decreasing order of $N_{\mathrm{WR}}$ (column 19). The electronic version contains data for all the 567 fibres, arranged in the increasing order of fibre number (column 1). The note to the table contains a detailed explanation of the quantities in each column. In Fig. A1, we show a spaxel map of MEGARA, where we indicate the location of each fibre, identified by its number. 
Table A1. He II $\lambda 4686$ nebular and WR parameters measured in individual fibre spectra

\begin{tabular}{|c|c|c|c|c|c|c|c|c|c|c|c|c|c|c|c|c|c|c|c|c|c|}
\hline \multirow[b]{2}{*}{ Fno } & \multicolumn{3}{|c|}{ Fibre coordinates } & \multirow[b]{2}{*}{$\begin{array}{r}\mathrm{PA} \\
0\end{array}$} & \multicolumn{4}{|c|}{$\mathrm{H} \beta$-related } & \multicolumn{2}{|c|}{$\mathrm{H} \gamma$-related } & \multicolumn{5}{|c|}{ HeII $\lambda 4686$ nebula-related } & \multicolumn{4}{|c|}{ HeII $\lambda 4686$ WR-related } & \multicolumn{2}{|c|}{ Extinction } \\
\hline & $\begin{array}{r}\mathrm{dX} \\
\operatorname{arcsec}\end{array}$ & $\begin{array}{r}d Y \\
\operatorname{arcsec}\end{array}$ & $\begin{array}{r}R \\
p c\end{array}$ & & $f_{T}$ & snr & $\begin{array}{r}\mathrm{EW} \\
\AA\end{array}$ & $\begin{array}{r}\mathrm{FW} \\
\AA\end{array}$ & $\mathrm{f} \beta$ & snr & $\begin{array}{c}\lambda_{0} \\
\AA\end{array}$ & $\begin{array}{r}\mathrm{FW} \\
\AA\end{array}$ & $f_{T}$ & snr & $\mathrm{f} \beta$ & $\begin{array}{r}\lambda_{0} \\
\AA\end{array}$ & $\begin{array}{r}\mathrm{FW} \\
\AA\end{array}$ & $N_{\mathrm{WR}}$ & $\mathrm{e} N$ & $\begin{array}{l}A_{\mathrm{V}} \\
\mathrm{mag}\end{array}$ & $\begin{array}{l}\mathrm{e} A_{\mathrm{V}} \\
\mathrm{mag}\end{array}$ \\
\hline 1 & & & 4 & 5 & 6 & 7 & 8 & 9 & 10 & 11 & 12 & 13 & 14 & 15 & 16 & 17 & 18 & 19 & 20 & & \\
\hline 242 & 1.86 & -1.61 & 36.9 & 229.1 & 6.181 & 82.7 & 51.2 & 1.2 & 19.6 & 10.8 & 4685.1 & 4.1 & 10.230 & 8.8 & 3.7 & 0.0 & 0.0 & 0.0 & 0.0 & 4.52 & 0.43 \\
\hline 240 & 2.32 & 34 & 40.3 & 0.0 & 990 & 56.2 & 41.1 & 1.3 & 19.6 & 9.1 & 4684.7 & 2.5 & 8.726 & 10.7 & 4. & 0.0 & 0.0 & .0 & 0.0 & 4.40 & 0.48 \\
\hline 244 & 2.32 & -1.88 & 44.9 & 1.0 & 972 & 59.9 & 57.1 & 1.2 & 21.8 & 9.6 & 4684.9 & 2.9 & 6.808 & 9.6 & 3 & 0.0 & 0.0 & 0.0 & 0.0 & 4.12 & 0.48 \\
\hline 246 & 1.86 & -2.15 & 42.7 & 220.8 & 3.010 & 70.5 & 64.5 & 1.2 & 23.4 & 16.7 & 4684.8 & 2.1 & 4.168 & 10.3 & 3.1 & 0.0 & 0.0 & 0.0 & 0.0 & 3.65 & 0.25 \\
\hline 406 & 0.46 & -4.03 & 60.9 & 186.6 & 2.171 & 67.0 & 50.5 & 1.3 & 23.0 & 14.1 & 4685.1 & 2.1 & 2.881 & 9.2 & 0.1 & 0.0 & 0.0 & 0.0 & 0.0 & 3.71 & 0.30 \\
\hline 20 & -7.89 & 0.81 & 119.2 & 84.2 & 2.144 & 63.4 & 41.1 & 1.4 & 24.2 & 10.4 & 4684.8 & 1.4 & 1.503 & - & & 0.0 & 00 & & 0 & & .45 \\
\hline 248 & 2.32 & -2.42 & 50.4 & 23.8 & .140 & 3.2 & 61. & 1.2 & 3.4 & 13. & 884. & 1. & .25 & & & & & & & & .34 \\
\hline 256 & 2.79 & -1.61 & 48.4 & 10.0 & 867 & 61.4 & 39.4 & 1.4 & 21.8 & $\begin{array}{r}8.9 \\
\end{array}$ & 4685.1 & 2.4 & 3.300 & 7.5 & 3 & 0.0 & 0.0 & 0.0 & 0.0 & 3.85 & 0.51 \\
\hline 250 & 1.86 & -2.68 & 49.1 & 14.7 & 1.791 & 79.6 & 68.2 & 1.2 & 25.3 & 12.8 & 4684.8 & 1.7 & 1.621 & 7.4 & & 0.0 & 0.0 & 0.0 & 0.0 & 3.20 & 0.36 \\
\hline 234 & 1.39 & -2.42 & 41.9 & 210.0 & 1.667 & 80.9 & 59.2 & 1.3 & 25.6 & 15.2 & 4684.7 & 2.0 & 2.191 & 10.9 & 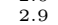 & 0.0 & 0.0 & 0.0 & 0.0 & 3.19 & 0.29 \\
\hline 260 & 2.79 & -2.15 & 52.9 & 32.4 & 1.450 & 51.2 & 44.5 & $\begin{array}{l}1.5 \\
1.4\end{array}$ & 23.4 & 9.4 & 4684.6 & $\begin{array}{l}2.0 \\
2.4\end{array}$ & $\begin{array}{l}1.845 \\
\end{array}$ & 6.0 & 2 & 0.0 & 0.0 & .0 & 0.0 & 3.55 & .46 \\
\hline 238 & 1.39 & -2.95 & 49.1 & 5.2 & 1.290 & 87.0 & 61.5 & 1.3 & 268 & 16.4 & 4685.0 & 2.5 & 1.594 & a & & 0.0 & & & & 2.93 & 0.27 \\
\hline 230 & 1.39 & -1.88 & 35.2 & 6.5 & 1.088 & 67.2 & 42.1 & 1.3 & 26.0 & 13.6 & 884.8 & 2.8 & 2.052 & 11.3 & 4 & 0.0 & 0.0 & 0.0 & 0.0 & 03 & .32 \\
\hline 497 & 3.25 & -0.27 & 49.0 & 5.3 & 1.033 & 49.4 & 28.6 & 1.8 & 23.4 & 10.7 & 0.0 & 0.0 & 0.000 & 0.0 & & 0.0 & 0.0 & 0.0 & 0.0 & 3.46 & 0.39 \\
\hline 468 & 1.86 & -1.07 & 32.2 & 40.0 & 0.997 & 49.7 & 26.3 & 1.5 & 24.2 & 9.3 & 4685.1 & 2.6 & 1.294 & 5.4 & 2 & 0.0 & 0.0 & 0.0 & 0.0 & 3.31 & 0.47 \\
\hline $\begin{array}{l}372 \\
372\end{array}$ & $\begin{array}{l}1.86 \\
1.86\end{array}$ & $\begin{array}{l}-3.22 \\
\end{array}$ & 55.9 & 10.0 & 0.986 & 64.6 & 60.4 & 1.2 & 27.3 & 20.6 & 4684.8 & 1.7 & 0.915 & $\begin{array}{r}3.4 \\
10.4\end{array}$ & & 0.0 & 0.0 & .0 & 0.0 & 2.79 & 0.18 \\
\hline 374 & 2.32 & 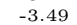 & 63.0 & 213.6 & .946 & 54.0 & 50.0 & 1.2 & 25.4 & 11.9 & 4684.3 & & & & & 0.0 & & & & & .36 \\
\hline 404 & 0.3 & -3.76 & 5 & & & & & 1.3 & 2 & 9 & & 2.3 & & & & & & & & & 47 \\
\hline $\begin{array}{l}404 \\
358\end{array}$ & 2.79 & -3.22 & 64.0 & 220.8 & .896 & 47.0 & 47.2 & $\begin{array}{l}1.0 \\
1.2\end{array}$ & $\begin{array}{l}24.9 \\
25.2\end{array}$ & 9.0 & 884.8 & $\begin{array}{l}2.0 \\
2.3\end{array}$ & 0.850 & 4.8 & 2.1 & 0.0 & 0.0 & .0 & 0.0 & 3.21 & 49 \\
\hline 513 & 3.71 & -0.54 & 56.4 & 1.8 & .869 & 51.4 & 26.4 & 1.7 & 23.9 & 8.8 & 0.0 & 0.0 & 0.000 & 0.0 & 0 & 0.0 & 0.0 & .0 & 0.0 & 3.26 & 0.49 \\
\hline 615 & 0.31 & -8.59 & 129.1 & 2.1 & 0.846 & 55.7 & 78.0 & 1.3 & 28.0 & 8.8 & 0.0 & 0.0 & 0.000 & 0.0 & & 0.0 & 0.0 & .0 & 0.0 & 2.77 & 0.53 \\
\hline 236 & 0.93 & $\begin{array}{l}-0.05 \\
-2.68\end{array}$ & $\begin{array}{r}42.7 \\
\end{array}$ & 199.1 & 0.843 & 70.3 & 44.7 & $\begin{array}{l}1.5 \\
1.4\end{array}$ & 27.2 & $\begin{array}{r}0.0 \\
15.2\end{array}$ & 684.8 & $\begin{array}{l}0.0 \\
2.5\end{array}$ & $\begin{array}{l}1.393 \\
1.393\end{array}$ & 11 & & 0.0 & & & & & .28 \\
\hline 264 & 2.79 & -2.68 & 58.1 & & & 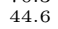 & & 12 & 20 & 11. & & & & & & & & & & & 36 \\
\hline 232 & 0.93 & -2.15 & 35.2 & 203.4 & 832 & 70.3 & 29.7 & 1.5 & 4.9 & 9.5 & 4.8 & 1.1 & .584 & 4. & 1. & 85.0 & & 7 & 0.1 & 08 & .48 \\
\hline 390 & 0.93 & $\begin{array}{l}-3.76 \\
\end{array}$ & 58.2 & 193.9 & 0.773 & 77.1 & 48.8 & 1.4 & 27.5 & 15.1 & 4685.2 & 2.0 & 0.822 & 8.7 & 2.4 & 0.0 & 0.0 & 0.0 & 0.0 & 2.76 & 0.29 \\
\hline 18 & 0 & 0.31 & 7 & ) & 175 & 320 & 2.3 & 2. & 18.9 & 5. & 0 & 0.0 & j & 0.0 & & .5 & 8 & & & 6 & 43 \\
\hline 416 & & & 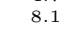 & & & & & & & & & & & & & & & & & & \\
\hline 420 & 0 & -0.27 & 8.1 & 0.0 & 42 & 4 & 2 & 2.2 & 1 & 4. & 84.5 & 1. & 0.326 & 3 & 5. & 6.3 & 5.6 & 3 & 0.8 & 1.10 & 0.54 \\
\hline 414 & & & 8.1 & 0.0 & & & 2 & 2.1 & & 5 & 0.0 & & & & & & 9.8 & & 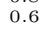 & & 0.51 \\
\hline 419 & -7.43 & & 111.6 & 8 & & 58.5 & 11.2 & 1.4 & 22.2 & 14.6 & 4685.0 & 2.2 & & & & & 73.1 & 11.2 & 0.6 & 1.87 & 9 \\
\hline $\begin{array}{l}415 \\
422\end{array}$ & 0.31 & -0. & 8.1 & 21 & & $\begin{array}{l}37.2 \\
37.2\end{array}$ & & $\begin{array}{l}2.4 \\
2.3\end{array}$ & 24.7 & 5 & & & & & & & 24.9 & 0 & & 0 & 43 \\
\hline 144 & -0 & & 8 & & & & & & & & & 4 & & & 12 & & & & & & .00 \\
\hline 446 & & & & & & & & & & & & & & & & & & & & & .51 \\
\hline 21 & & & & & & & 3 & 2. & & & & & & & & & 24 & & & & 49 \\
\hline 148 & & & 8 & & & & & & & & & & & & & & & & & & 55 \\
\hline 424 & & & & & & & & & & & & & & & & & 2 & 4. & 0 & 0 & .35 \\
\hline $\begin{array}{l}424 \\
442\end{array}$ & & & & & & & & & & & 4 & & & & & & & & & & .55 \\
\hline $\begin{array}{l}44 \\
14\end{array}$ & & & & & & & & & & & & & & & & & & & & & \\
\hline 15 & -0 & -0 & & 15 & & & & & & & & & & & & & & & & & 0 \\
\hline 14 & & & & & & & & & & & & & & & & & & & & & \\
\hline 53 & & & & & & & & & 13 & & & & & & & & & 2. & & & \\
\hline 149 & -2.7 & -0.54 & 42.6 & 100 & & 12.9 & 1 & 2 & 9 & 1. & 468 & 2 & & 0 & 9 & & 2 & 2. & 0.3 & 0 & 0.00 \\
\hline 423 & & -0 . & 11 & & & & 10 & & & & & & & & & & & & & & 39 \\
\hline 19 & & & & & & & & & & & & & & & & & & & & & \\
\hline 41 & & & & & & & & & & & & & & & & & & & & & \\
\hline 51 & & & & & & & & & & & 5 & & & & & & & 8 & & & 96 \\
\hline 448 & 1. & -0.27 & 21.3 & 259.1 & .109 & 42. & 8.1 & 1.7 & 27.5 & 7.3 & 0.0 & 0 & 0 . & 0 & 0 & 9.4 & 25.0 & 1.8 & 0.3 & 1.91 & 0.54 \\
\hline $\begin{array}{l}440 \\
452\end{array}$ & 1.3 & -0.81 & 24.2 & 240 & & 49 & $\begin{array}{r}0.1 \\
12.2\end{array}$ & 1.7 & 25 & 9 & 0.0 & 0 & & & & & 2 & & 0.2 & $\begin{array}{l}1.51 \\
2.58\end{array}$ & 0.43 \\
\hline 213 & & & & & & & & & & & & & & & & & & & & & \\
\hline 49 & 5.57 & & 7.1 & $\begin{array}{r}73.9 \\
73.9\end{array}$ & & 8.4 & 11.6 & 2. & 1.8 & 3.8 & 5.4 & 0. & & 2.6 & 2.4 & & 2.7 & 1.4 & 0.3 & $\begin{array}{l}1.63 \\
1.63\end{array}$ & 8 \\
\hline
\end{tabular}

Row description:

Data for 50 fibres, ordered according to the decreasing contribution in $\mathrm{H} \beta$ (column 6 ) in the first 25 rows, and ordered according to the decreasing number of WR stars (column 19) in the next 25 lines. Table containing data for all 567 fibres (excluding the sky fibres) is available in the electronic version.

Column description:

1 - Fibre number.

2-3 - X and Y shifts in arcsecs with respect to (wrt) the core of SSC-A, which is the closest to fibre \#418. Objects to the east and south of SSC-A have negative shifts.

4 - distance from SSC-A in parsec using a scale of $15 \mathrm{pc} \operatorname{arcsec}^{-1}$.

5 - PA in degrees of the fibre position wrt SSC-A.

$6-100 \times$ the extinction-corrected $\mathrm{H} \beta$ flux in the fibre wrt total $\mathrm{H} \beta$ flux $\left(4.444 \times 10^{-12} \mathrm{erg} \mathrm{cm}^{-2} \mathrm{~s}^{-1}\right)$.

7 - Signal-to-noise ratio (SNR) of the $\mathrm{H} \beta$ line (see equation 1 ).

8 - Emission Equivalent Width $(\mathrm{EW})$ in $\AA$ of the $\mathrm{H} \beta$ emission line.

9 - FWHM in $\AA$ of the fitted Gaussian to the emission line of $\mathrm{H} \beta$.

$10-100 \times \frac{f_{H \gamma}}{f_{H \beta}}$ ratio.

11 - SNR of the $\mathrm{H} \gamma$ emission line.

12 - Central wavelength in $\AA$ of the fitted He II $\lambda 4686$ narrow (nebular) line.

13 - FWHM in $\AA$ of the fitted He II $\lambda 4686$ narrow (nebular) line.

$14-100 \times$ the extinction-corrected HeII $\lambda 4686$ nebular line flux in the fibre wrt total flux in the same line $\left(9.13 \times 10^{-14} \mathrm{erg} \mathrm{cm}^{-2} \mathrm{~s}^{-1}\right)$.

15 - SNR of the fitted He II $\lambda 4686$ narrow (nebular) line.

$16-100 \times \frac{f_{H e i i \lambda 4686(\text { nebular })}}{f_{H \beta}}$ ratio.

17 - Central wavelength in $\AA$ of the fitted He II $\lambda 4686$ broad (WR) line.

18 - FWHM in $\AA$ of the fitted He II $\lambda 4686$ broad (WR) line.

19 - Luminosity of the He II $\lambda 4686$ broad line expressed in units of $1.22 \times 10^{36} \mathrm{erg} \mathrm{s}^{-1} \equiv N_{\mathrm{WR}}=$ equivalent number of WNL stars.

20 - Error in $N_{\mathrm{WR}}$, determined by propagating errors in the He II $\lambda 4686$ broad line flux.

$21-A_{\mathrm{V}}$ determined from $\mathrm{H} \gamma$ and $\mathrm{H} \beta$ lines when both the lines have $S N R>3$ (absorption correction $1 \AA$ in EWs.)

22 - Error on $A_{\mathrm{V}}$, determined by propagating errors in the $\mathrm{H} \beta$ and $\mathrm{H} \gamma$ line fluxes. 


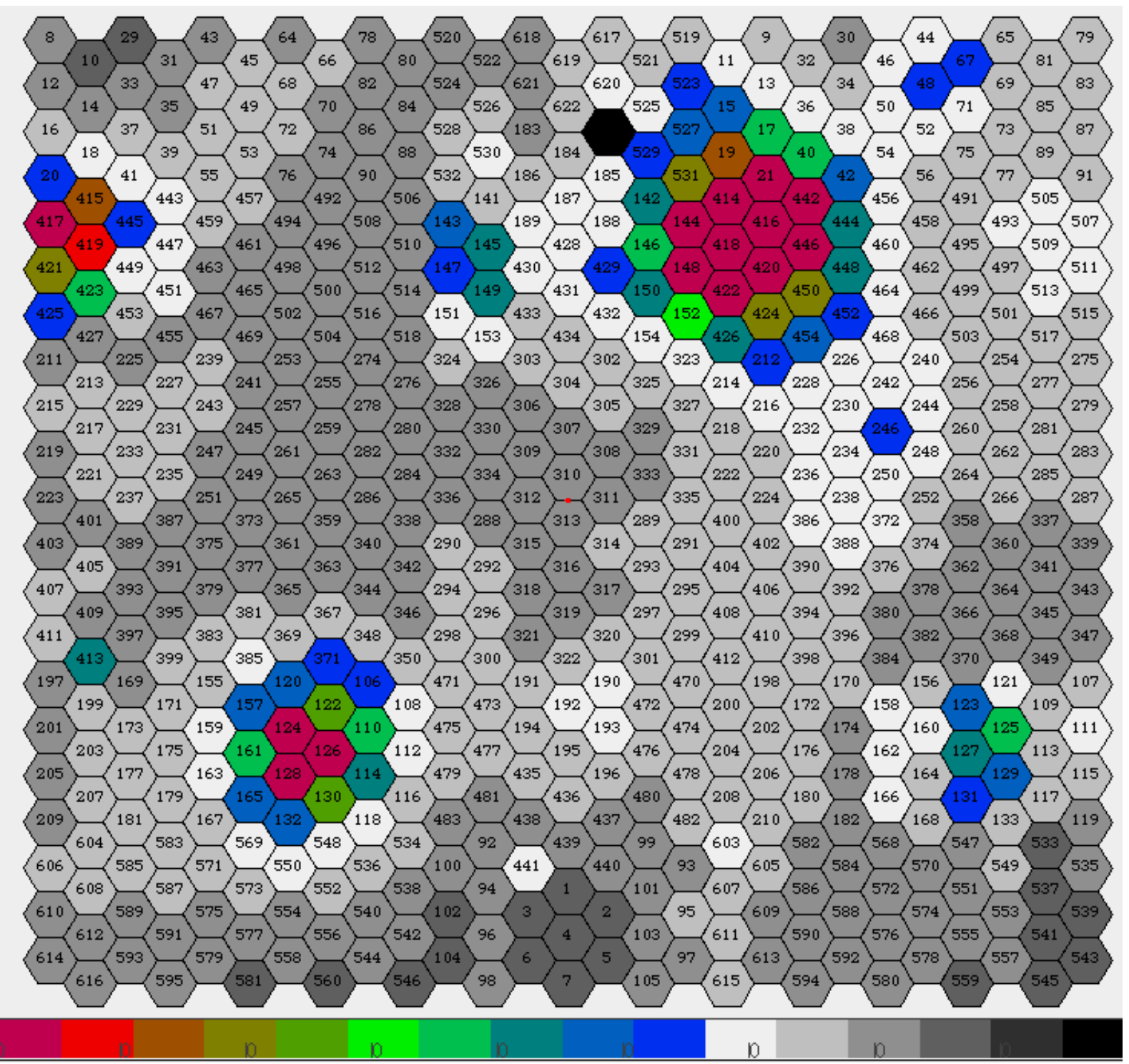

Figure A1. Spaxel (hexagon) map of the region of NGC 1569 observed by MEGARA. The spaxel contains the summed flux from the entire spectra. The colour-scale at the bottom: the black colour indicates faint regions and the red the brightest regions. The numbers indicate the fibre identification numbers. The fibres nearest to the centres of SSC-A and SSC-B are \#418 and 128, respectively. All coloured hexagons in the same rows but to the left of SSC-A, and to the right of SSC-B are artefacts due to the cross-talk with the fibres pointed to these two bright clusters, respectively. 